\title{
The impact of Paleocene/ Eocene (P/E) Thermal Maximum (PETM) of some sections in Sinai - Egypt, based on benthonic foraminifera
}

\author{
Ayyad, S. N.*, H. El Nadi**, M. A. Omran*** and H. F. Abd Allah** \\ * Geology Dept., Faculty of Science, Mansoura University, Mansoura, Egypt \\ ** Geological and Biol. Sci. Dept., Suez Canal Univ., El-Arish, Faculty of Education, El-Arish, North Sinai, Egypt. \\ *** Geology Dept., Faculty of Sci., Suez Canal University, Ismailia, Egypt.
}

\section{Received: $7 / 11 / 2013$}

\begin{abstract}
The present study deals with lithostratigraphy, biostratigraphy and paleoecology of paleocene - Early Eocene benthonic foraminifera northeast Sinai, Egypt; G. El Falig, G. El Qusaima, G. Holet Abu Senna and G. Um Thamalla have been collected, sampled and studied based on benthonic foraminifera. The measured sections comprises; Esna Formation and Thebes Formation. The exposed rock units contain rich and high diverse assemblage of benthonic foraminifera that are discussed. They yield 109 foraminiferal benthonic species belonging to 48 genera, 24 subfamilies, 25 families, 18 super families, 3 suborder and one order. The investigation of the foraminiferal content led to the identification of two assemblage zones; the Early - Late Paleocene Neoflabellina - Spiroplectammina Gavelinella Assemblage Zone and Late Paleocene - Early Eocene Loxostomoides - Cibicidoides - Bulimina Assemblage Zone. During the investigated interval three biofacies with three faunal turnover events have been identified in the studied area reflecting a major sea level change. These turnovers are characterized by discontinuous distribution of species, changes of the relative abundance of species and diversification. The Esna Shale was deposited in middle neritic to upper bathyal environment and Thebes Formation was also deposited in progressively shallowing middle neritic environment.
\end{abstract}

Keywords: Benthonic foraminifera, Lithostratigraphy, Biostratigraphy, Paleoecology, Turnover, Biofacies, Paleoenvironment, PETM, BFEE, Paleocene, Eocene, Sinai, Egypt.

\section{INTRODUCTION}

For the benthonic foraminifera; the $\mathrm{P} / \mathrm{E}$ boundary event has been regarded as the largest turnover in the deep-sea benthic foraminifera during the last million years (Tjalsma \& Lohmann, 1983; Shahin, 2001). The event is marked by extinction of many intermediate and deep-water species more than one third of the taxa: $33-50 \%$ per site, as well as some shallow dwelling (50to $150-\mathrm{m}$ water depths) benthic foraminifera $(22 \%$; Kaiho, 1994b). Although changes in deep-sea benthic foraminifera test size and an oxygen index have been established at low resolution for the past 120 m.y. (Kaiho, 1994b, 1999a), these indices have yet to be applied at high resolution to the $\mathrm{P} / \mathrm{E}$ boundary. The parameters control the composition and size range of shallow-water benthic foraminiferal assemblages was the food resources and algal symbiosis (Hallock, 1985). The deep-sea benthonic foraminifera affected by factors such as dissolved oxygen levels and food supply (Phleger \& Soutar, 1973; Machain 1984; Kaiho, 1994a, 1998, 1999a).

Sinai Peninsula is an important part of the southern Tethys and coincides with a belt of wind-induced upwelling (Parrish \& Curtls, 1982). Several authors in Sinai have intensively worked out the P/E boundary. Among the recent studies, there are, (Marzouk \& Luning, 1998; Luning et al. 1998; Obaidalla, 1999; Shahin, 1998, Ayyad, 2001; El-Nady \& Shahin, 2001; El-Nady, 2002; Ayyad et al., 2003; Faris et al., 2005; El-Nady, 2005, 2008; Morsi et al., 2008).

Four sections have been measured and studied in details during the Paleocene/Eocene boundary interval in northeastern Sinai Peninsula; around El Qusaima city and namely; G.El-Falig (30³7' 30" N, 34 $\left.10^{\circ} 10^{\prime} 26^{\prime \prime} \mathrm{E}\right)$;
G.Um Thamalla $\left(30^{\circ} 38^{\prime} 18^{\prime \prime} \mathrm{N}, 33^{\circ} 53^{\prime} 12^{\prime \prime} \mathrm{E}\right)$; Holet

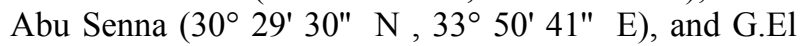

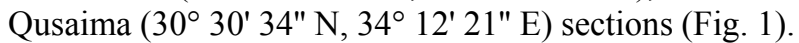

Out of 381 samples were collected from the studied area representing the four sections subdivision as follow, (85) samples from G.El-Falig section, (104) samples from G.Um Thamalla section, (111) samples from G.Holet Abu Sena section and (81) samples from G.El Qusaimaa section prepared for the foraminiferal .

Nearly about 110-120 gram of dried rock was impregnated with a soda solution and washed after several days and washed over a $0.063 \mu \mathrm{m}$ sieve. This process was repeated until the residues were clean.

The samples picked up by special brush and examined using light binocular microscope. Photographs of the identified species are obtained using Scanning Electron Microscope (SEM).

\section{LITHOSTRATIGRAPHY}

The Paleocene - Lower Eocene sequences in G. El Falig, G. Um-Thamalla, G.El Qusima, and G. Holet Abu -Senna (2), northeastern Sinai were studied in the present work. These successions classified from older to younger into Esna and Thebes formations.

The Esna Formation: in all the studied sections, can be subdivided into three main parts as follow:

The Lower part (a); the base of this part is unexposed in the studied area, it is consists of hard to moderately hard, yellowish to dark grey Shale, marl, grey marl, gypsum vienlets and calcareous clay ranging in age from the Early to Late Paleocene. This part attains a thickness of about $43 \mathrm{~m}$ in G. Holet Abu Senna, $37 \mathrm{~m}$ at G.Um Thamalla , 30m in G. El Qusaima and about $31 \mathrm{~m}$ at G. El Falig . 


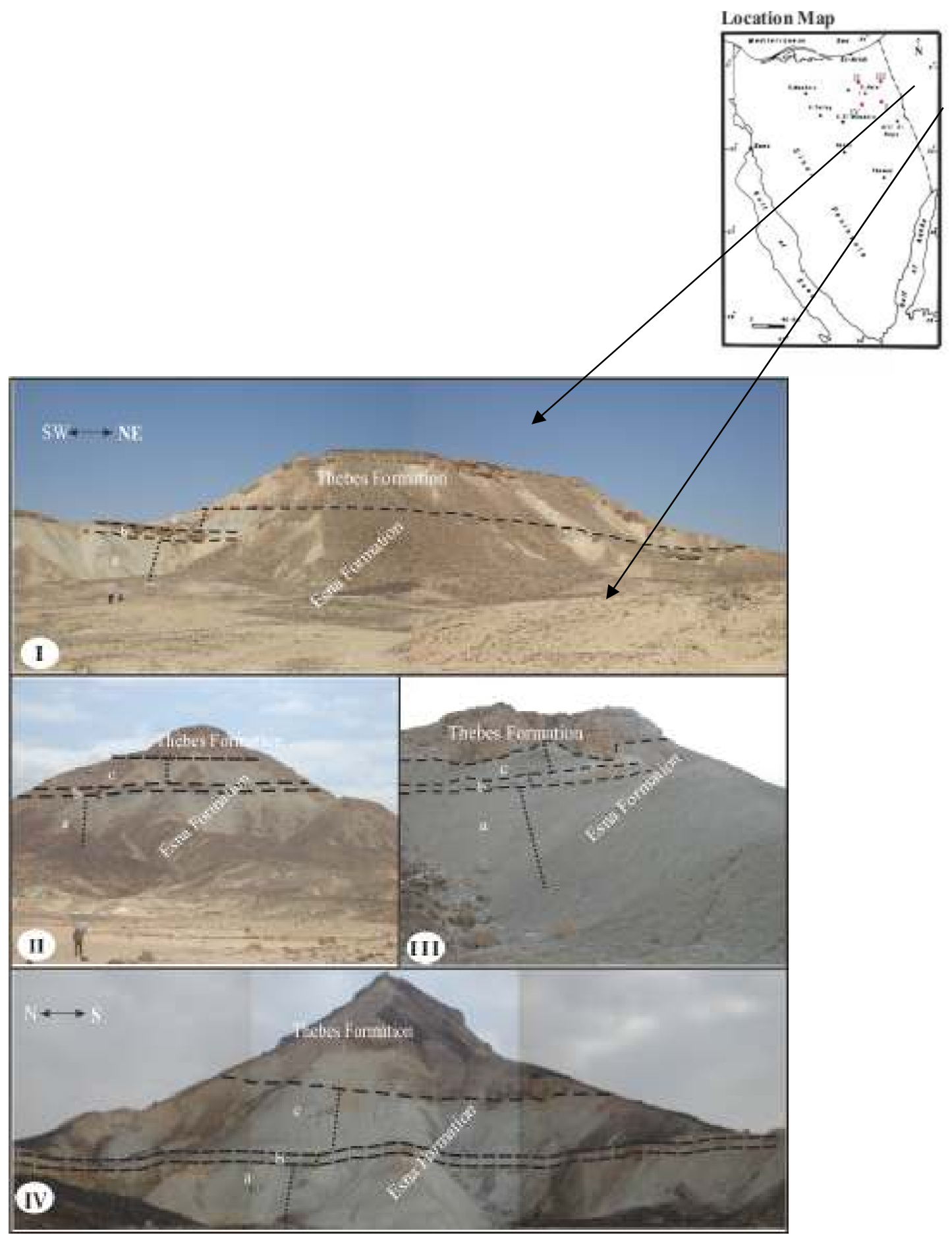

Fig (1): Location map: I- Gabal El Qusaima II- Gabal Holet Abu Senna III- Gabal El Falig IV- Gabal Um Thamalla

Middle part (b); this part represented by white chalky limestone, yellowish, and argillaceous limestone of Late Paleocene age. The thickness of this part in the studied area ranging from about, $4 \mathrm{~m}$ at $\mathrm{G}$. Holet $\mathrm{Abu}$ Senna, $3.5 \mathrm{~m}$ at G. Um Thamalla and nearly about $3 \mathrm{~m}$ at both G. El Falig and G. El Qusaima . This part is barren of microfossilis in all studied sections, equivalent to Tarawan Chalk (Kuss \& Marzouk, 2001).

Upper part (c); It is composed of blocky to fissile , moderately hard, light grey and calcareous Shale interceded with thin beds of marl. It has a thickness of about $15 \mathrm{~m}$ in G. Holet Abu Senna, 15.5 at G. El Qusaima, 12.5 at G. Um Thamalla and $13.5 \mathrm{~m}$ at G. El Falig. It overlies the Middle limestome part and underlies the Thebes Formation .

Thebes Formation: This rock unit is recorded from all the studied sections and consist mainly of yellowish to greenish marl, interbeded with chalky limestone, brownish flint nodules and concretions. The upper part of this formation is not reached in the studied sections . 
It belong to the Early Eocene age and conformably overlies the Esna formation.

\section{BIOSTRATIGRAPHY}

\section{Benthonic Foraminiferal Zonation:}

In the present study, the stratigraphic distribution of the identified benthonic foraminiferal species and varities from the Paleocene - Early Eocene of the measured successions allowed to subdividing this interval into tow assemblage benthonic foraminiferal zones. Their age was determined by the equivalent planktonic foraminiferal zones. Their evolution and stratigraphic distribution were recorded from different regions in and outside Egypt by several authors ilustrated in Table (8). The proposed biostratigraphic zones with their stratigraphic position and its age briefly discussed from base to top as follows:

\section{Neoflabellina - Spiroplectammina - Gavelinella Assemblage Zone: \\ Contemporaneous unit: This assemblage is equivalent to the Parasubbotina pseudobulloides, Praemurica trinidadensis, Praemurica uncinata, Morozovella angulata, and Igorina pusilla pusilla zones ( figure 2).}

Stratigraphic Position: This assemblage zone occupies the lower and middle parts of the Esna Shale. It covers $22.4 \mathrm{~m}$ in G. El Falig, $25.3 \mathrm{~m}$ in G.El Qusaima, $26.25 \mathrm{~m}$ in G.Holet Abu Senna and $21 \mathrm{~m}$ in G, Um thamalla.

Age assignement: Lower and middle Paleocene .

Assemblages: This assemblage zone is charactrized by abundances and most common taxa of Pullenia coryelli (White) and Gavelinella beccariiformis (White). In addition to the following taxa; Spiroplectimmina kenbeli (Le Roy), S. spectabilis (Grzybowski), Spiroplectinella dentata (Alth), Dentelnia basiplanata Cushman, D. catenula Reuss, D. colei Cushman \& Dusenbury, D. graacilis D'orbigny, D. megalopolitana Reuss, Verneuilina cretacea (Karrer), Neoflabellina jarvisi (Cushman), Astaculus munds (Cushman), Vaginulinopsis trilobata (D'orbigny), Hemirobulina bullata (Reuss), Dorothia pupa (Reuss), D. bulletta (Carsey), Gaudryina pyramidata Cushman, G. laevigala Franke, Pseudogaudryinella compacta. (Ten Dam \& Sigal), Stilostomella paleocenica (Cushman \& Todd), Pleurostomella clavata (Cushman), Coryphostoma platium (Carsey), Frondicularia cuspidata (Cushman), F. bidentata (Cushman), Vaginulinopsis trilobata (D'orbigny), Loxostomoides applinae (Plummer), Marssonella indentata (Cushman \& Jarvis), M. oxycona (Reuss), Ammodiscus cretaceous (Reuss), Anomalinoides midwayensis (Plummer), A. rubiginosus (Cushman), Quadrimorphina allomorphinoides (Reuss), Valvulina Colei Cushman, Clavulinoides asper (Cushman), Cibicides praecursorius (Schwager), Cibicidoides succedens (Brotzen), Cibicidoides alleni (Plummer), Cibicides ribbingi Brotzen, Angulogavelinella grands (Marssom), Nodosarella gracillima Cushman, Pseudoclavulina amorpha (Cushman), P. globulifera (Ten Dam \& Sigal), Ramulina globulifera (Brady), $R$. navarrona (Cushman), Lagena substriata (Williamson) ,
Lenticulina isidis (Schwager), L. midwayensis (plummer), Chilostomelloides cyclostoma (Rzehak), Ellipsopolymorphina velascoensis (Cushman).

Loxostomoides - Cibicidoides - Bulimina Assemblage Zone:

Contemporaneous unit: Globanomalina pseudomenardii, Morozovella velascoensis, M. edgari, M. Subbotina, M. formosa formosa, M. aragonensis and Acranina pentacamerata planktonic foraminiferal zones zones.

Stratigraphic position: This assemblage zone occupies the Upper part of the Esna Shale and the lower partof the Thebes Formation. The thickness of this zone attain of about $26.1 \mathrm{~m}$ in G. El Falig, $29.7 \mathrm{~m}$ in G. Um thamalla, $28.5 \mathrm{~m}$ in G.El Qusaima and $27.5 \mathrm{~m}$ in G.Holet Abu Senna.

Age assignement: Late Paleocene to Early Eocene .

Assemblages: This assemblage is charactrized by abundances and most common taxa of Cibicidoides succedens (Brotzen), C. praecursorius (Schwager), $C$. alleni (Plummer), Bulimina midwayensis (Cushman \& Parke), Loxostomoides applinae (Plummer), $L$. plummerae (Cushman). In addition to the following taxa; Lenticulina midwayensis (Plummer), Lenticulina isidis (Schwager), Gyroidinoides girardanus (Reuss), Dorothia pupa (Reuss), Anomalinoides welleri (Plummer), Coryphostomella telatynensis (GaworBiedowa), Dentelnia basiplanata Cushman, D. catenula Reuss, D. colei Cushman \& Dusenbury, D. graacilis D'orbigny, D. megalopolitana Reuss, Astaculus munds (Cushman), Vaginulinopsis trilobata (D'orbigny), Hemirobulina bullata (Reuss), Dorothia bulletta (Carsey), Gaudryina pyramidata Cushman, G. laevigala Franke, G. austinana Cushman, Pseudogaudryinella compacta. (Ten Dam \& Sigal), Marssonella indentata (Cushman \& Jarvis), M. trochus (D'orbigny), Ammodiscus cretaceous (Reuss), Anomalinoides midwayensis (Plummer), A. rubiginosus (Cushman), Vulvulina colei Cushman, Clavulinoides asper (Cushman), Cibicides ribbingi Brotzen, Angulogavelinella grands (Marssom), Nodosarella gracillima Cushman, Pseudodavulina amorpha (Cushman), Pseudoclavulina globulifera (Ten Dam \& Sigal), Ramulina globulifera (Brady), Ramulina navarrona (Cushman), Lagena substriata (Williamson)
Chilostomelloides cyclostoma (Rzehak),

Ellipsopolymorphina velascoensis (Cushman), Stilostomella paleocenica (Cushman \& Todd), Pleurostomella clavata (Cushman), Frondicularia cuspidata (Cushman), F. bidentata (Cushman), Vaginulinopsis trilobata (D'orbigny) and Quadrimorphina allomorphinoides (Reuss).

\section{PALEOECOLOGY AND PALEOBATHYMETRY ACROSS THE P/E BOUNDARY WITH SEA- LEVEL CHANGES}

The reconstruction of the water depth is an important factor to elucidate the paleoecology and the paleoproductivity across the Paleocene-Eocene $(\mathrm{P} / \mathrm{E})$ boundary. For this purpose, about $0.5 \mathrm{gm}$ of the residue 
of each sample has been used for quantitative foraminiferal parameters at the study section (Table 1). These parameters include the P/B ratio, the Aggl. /Calc. ratio, the total foraminiferal abundance and their diversification (Fig.7). During the normal condition, the percentage of planktonic foraminifera ( $\mathrm{P} / \mathrm{B}$ ratio) increases with water depth and depends mainly on the relative difference of paleoproductivity of foraminiferal assemblages (Murray, 1976, Shahin, 2001; El-Nady, $2005,2008)$. The changes in the relative abundance of these assemblages are used to interpret the rate and nature of the foraminiferal evolution and diversification across $\mathbf{P} / \mathbf{E}$ boundary. The productivity of benthic foraminiferal is higher in the shallow neritic environmental condition (Morkhoven et al., 1986;Kaiho et al., 2006; Shahin, 1992; 2001; El-Nady, 2005). In addition, the $\mathrm{P} / \mathrm{B}$ ratio can also be used as an environmental parameter of relative paleobathymetric changes and investigate the circulation pattern and current intensities of marginal seas to open ocean (Murray, 1976). However, the global cooling episode of the early Paleocene was followed a rapid warming episode during the P/E boundary (Shackleton \& Hall, 1985; Kennett \& Stott, 1990; Salis et al., 1998; Stott et al., 1991).

Highlights several events and striking changes in abundance and composition within the assemblages that represent paleoenvironmental events performed before/after and through (PETM). These changes clearly outline a complex ecological evolution across the (PETM). Which possible confined in three biofacies as follow;

\section{Biofacies 1( Pre-PETM):}

In the studied area, the benthic foraminiferal analysis seems that during that time span (before the boundary) appearance and disappearance of the species have been affected selectively in the habitants of the open marine realm. The time interval between the Early Paleocene Parasubbotina pseudobulloides to the Early Middle Paleocene Praemurica uncinata Zone is charactrized by adeep-water Velasco-fauna type, including the genera Gavelinella beccariiformis, Pullenia coryelli, Cibicides ribbingi, Spiroplectamina dentata, Anomalinoides rubiginosus and others, indicating an outer neritic environment. This interval represents the maximum marine transgressive events during the Early Paleocene in Sinai. Toward the Middle Paleocene Morozovella angulata Zone a noticeable drop in the foraminiferal parameters, so this time interval characterized by dominance of shallow Midway type-fauna with deep Velasco type-fauna represents the middle and outer neritic condition. This position continues to th end of Middle Paleocene Igorina pusillu pusilla Zone. In the Late Paleocene Globanomalina pseudomenardii Zone, the benthonic foraminiferl assemblage represented by outerr neritic deep Velascofauna type as Gavelinella beccariiformis and Pullenia coryelli , Tjalsma \& Lohmann (1983) and Speijer \& Van der Zwaan(1994), reported the same faunal assocition from Upper Middle-bathyal sediments.
Upward to the Late Plaeocene to the Morozovella velascoensis Zone, the Middle neritic environment prevailing over bathyal environment, and some species as, Gavelinella beccariiformis , Pullenia coryelli, disappear as indication for Middle neritic environment.

\section{Biofacies 2 (during - PETM):}

Coincident with PETM interval, benthonic foraminifera are on the contrary from planktonic foraminifera, where species richness reduce by approximately (30- 35\%) (Thomas, 1990; Thomas \& Shachleton, 1996; Oreshkina, 2003; Röhl et al., 2007; Alegret et al., 2009). In the studied sections, and during PETM, the extinition of benthonic foraminiferal taxa are charactrized this interval such as Anomalinoides rubiginosus, Palmula primativa, Coryphostoma midwayensis, Dorothia pupa, Chrysalogonium velascoense, Gyroidinoides girardanus, Marssonella oxycona, Neoflabellina jarvisi and Pullenia coryelli. Anoxic conditions at the sea-floor may have caused some extinctions locally, but not globally (e.g., Thomas, 2007). However, there is no agreement as to whether primary productivity in surface waters increased or decreased globally at the onset of the CIE (Bains et al., 2000; Bralower, 2002; Dickens et al., 2003; Tremolada $\&$ Bralower, 2004). The extinction of deep-sea benthonic foraminifera was global (Alegret et al , 2009). Moreover, food supply, oxygen conditions or dissolution seem not to be the cause of the extinctions, warming is likely to have been the only global feature of the PETM for which there was no refugia, triggering paleoecological and paleoenvironmental instability, and eventually leading to the gradual but rapid benthic extinction event (BEE) (Monechi et al., 2000 a,b). According to Berggren and Aubert (1975), representatives of the bathyal and abyssal Velasco-type fauna such as Cibicidoides velascoensis, Guadryina pyramidata, Dorothia bulletta, Anomalinoides rubiginosus and Coryphostoma midwayensis, are common at the studied area. In additioin, the prsence of Quadrimorphina sp., which are most common at abyssal depths (Tjalsma \& Lohmann, 1983), have been recorded. These data indicate outer neritic to Lower bathyal depth of deposition during the Late PaleoceneEarly Eocene PETM in the study sections.

\section{Biofacies 3 ( after - PETM):}

During the Late Paleocene interval, an important benthonic foraminifera (e.g. Tritaxia midwayensis, Entosolenia crebera, Coryphostoma midwayensis and Neoflabellina jarvisi) are completely disapeared. On the other hand, a new benthic species as (e.g. Cibicidoides libycus, C. simplex, Anomlinoides welleir and Bulinina midwayensis) has appeared. The presence of this midway-fauna type reflect open marine with Middle to outer neritic environment, upward to Morozovella aragonensis and Acarinina pentacamerata zones, covert to inner neritic environment at the end of Thebes Formation in the studied area (Fig.7). 


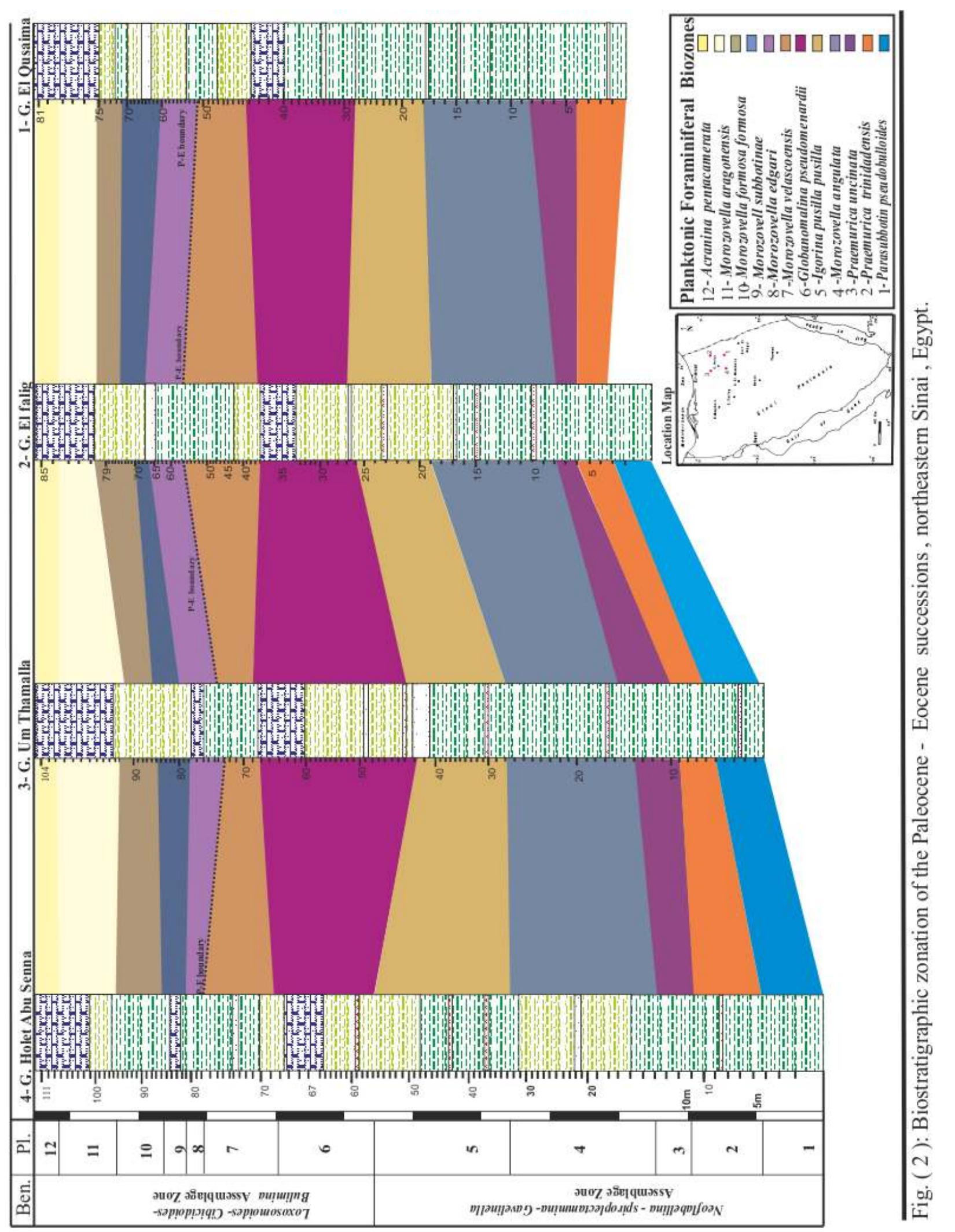




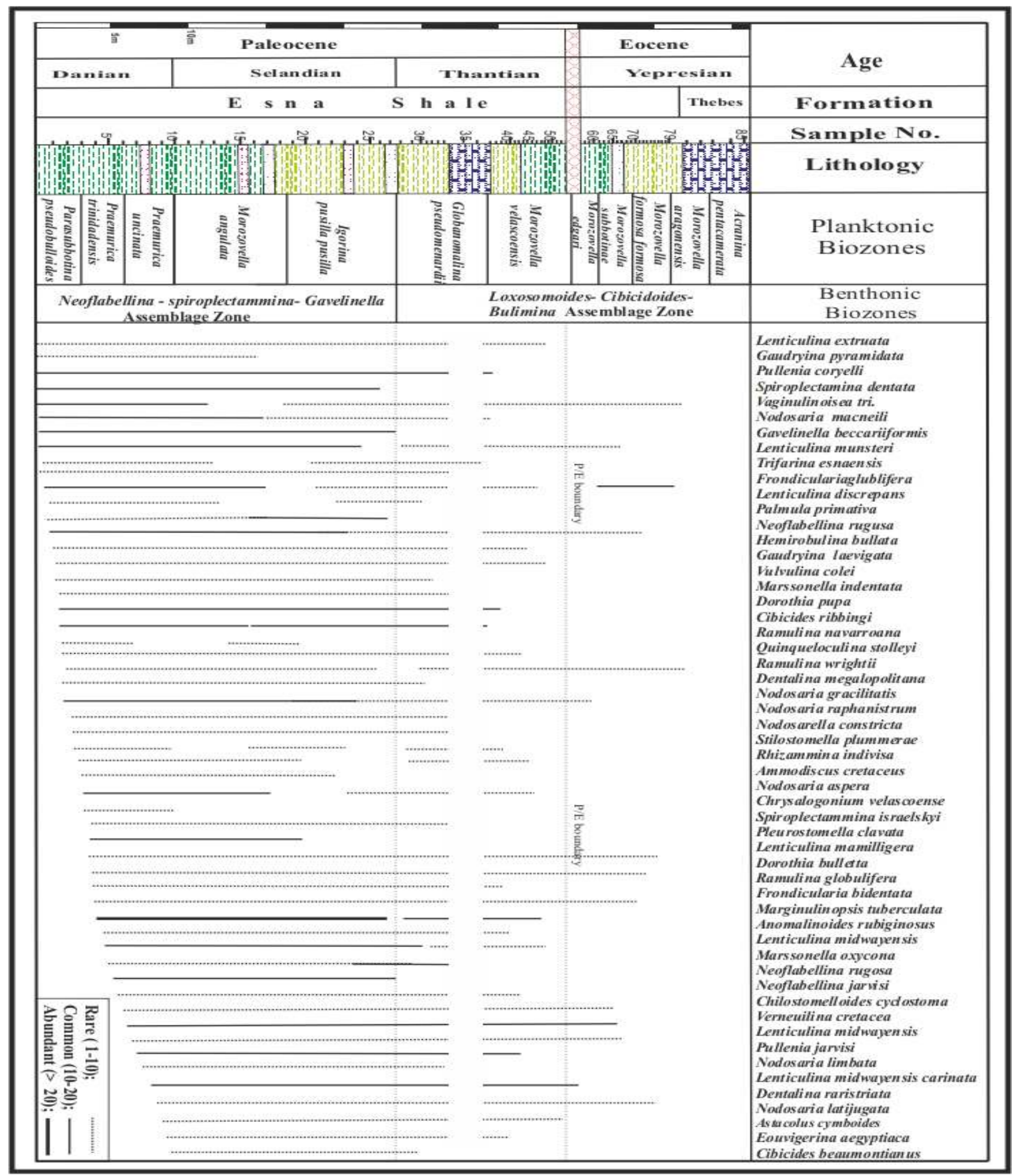

Fig. (3): Biostraigraphic chart of the benthonic foraminiferal species in G. El Falig section, northeastern Sinai, Egypt. 


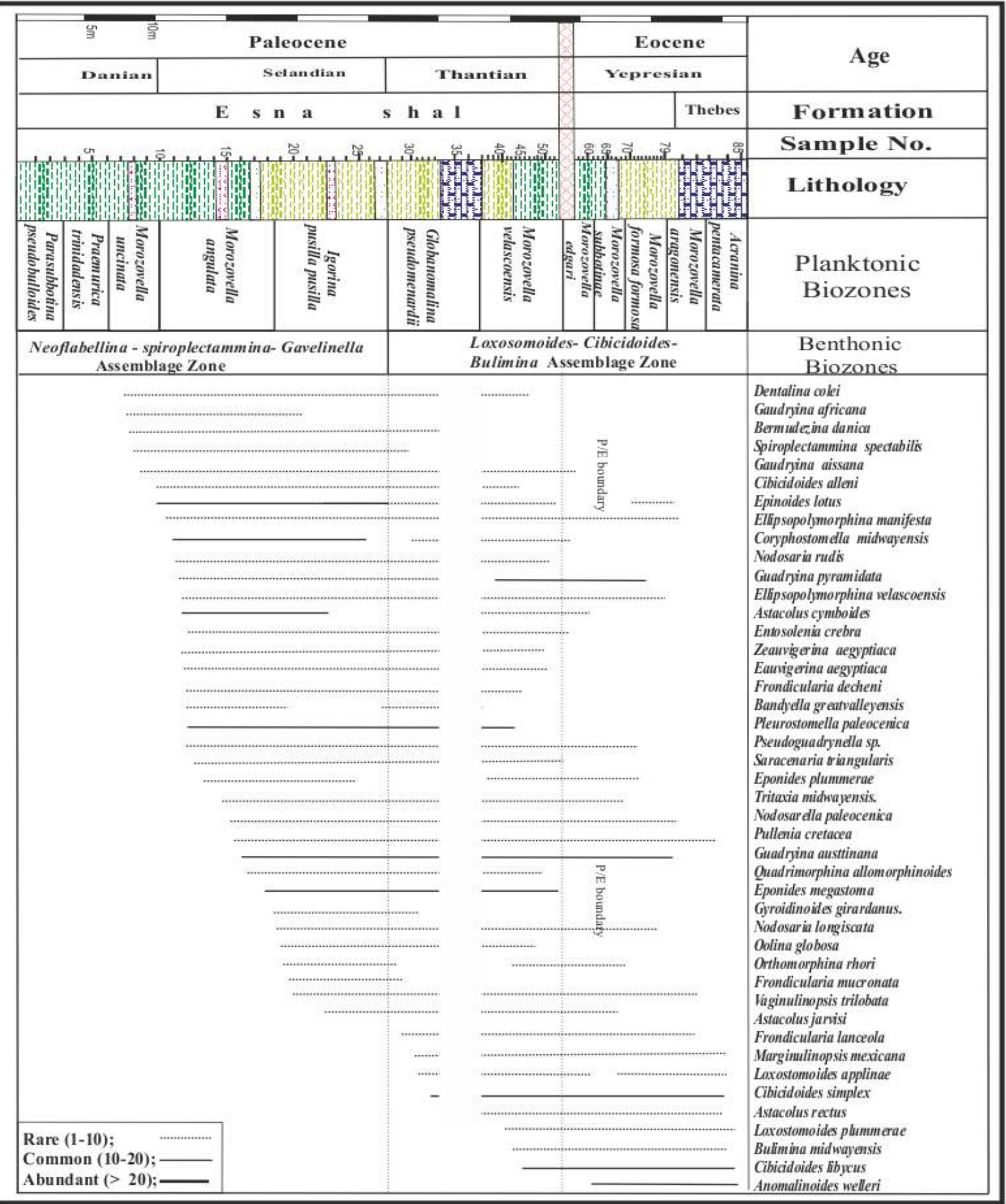

Fig. (3) Continued: Biostraigraphic chart of the benthonic foraminiferal species in G. El Falig section, northeastern Sinai, Egypt. 


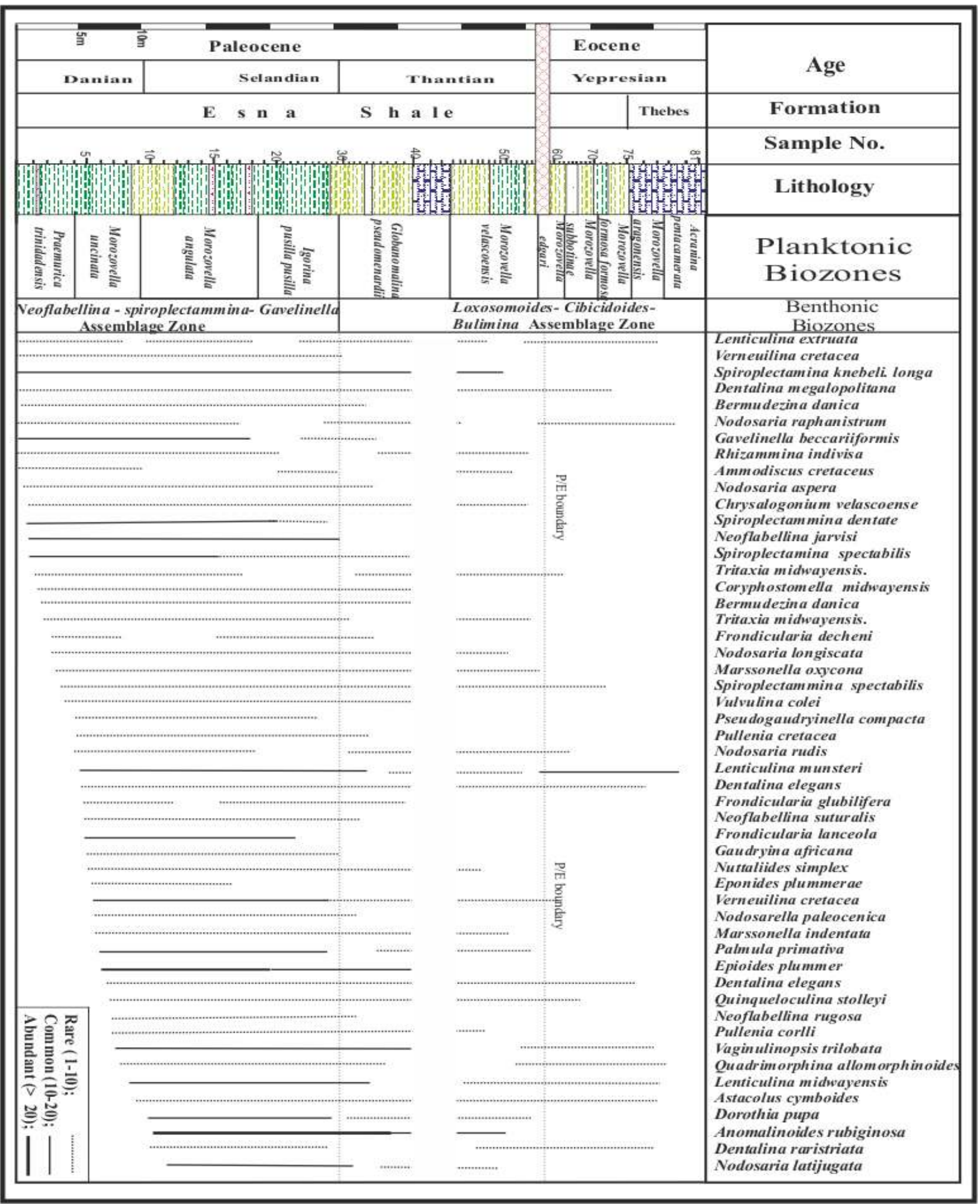

Fig. (4): Biostraigraphic chart of the benthonic foraminiferal species in G. El Qusaima section, northeastern Sinai, Egypt. 


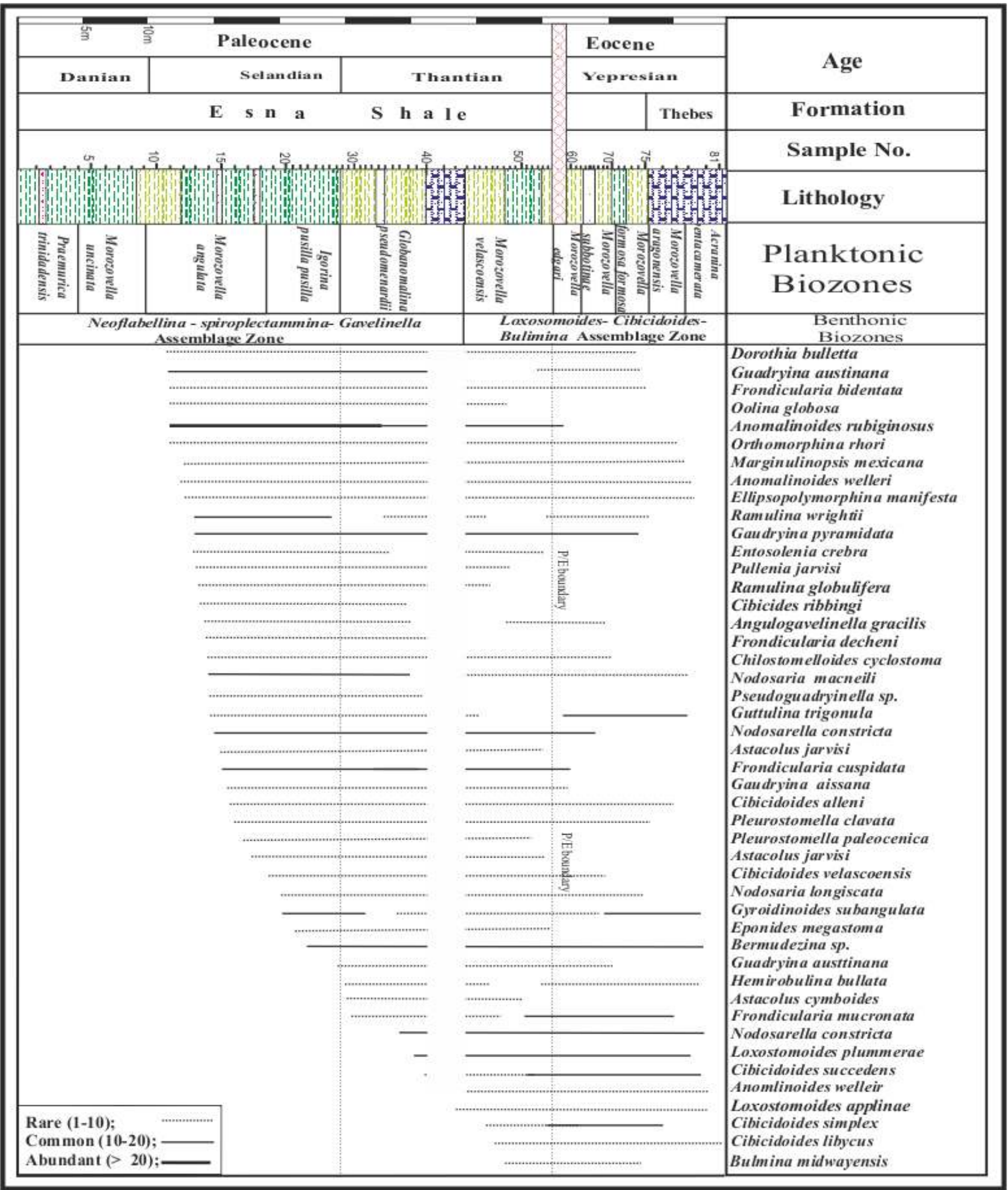

Fig. (4) Continue: Biostraigraphic chart of the benthonic foraminiferal species in G. El Qusaima section, northeastern Sinai, Egypt. 


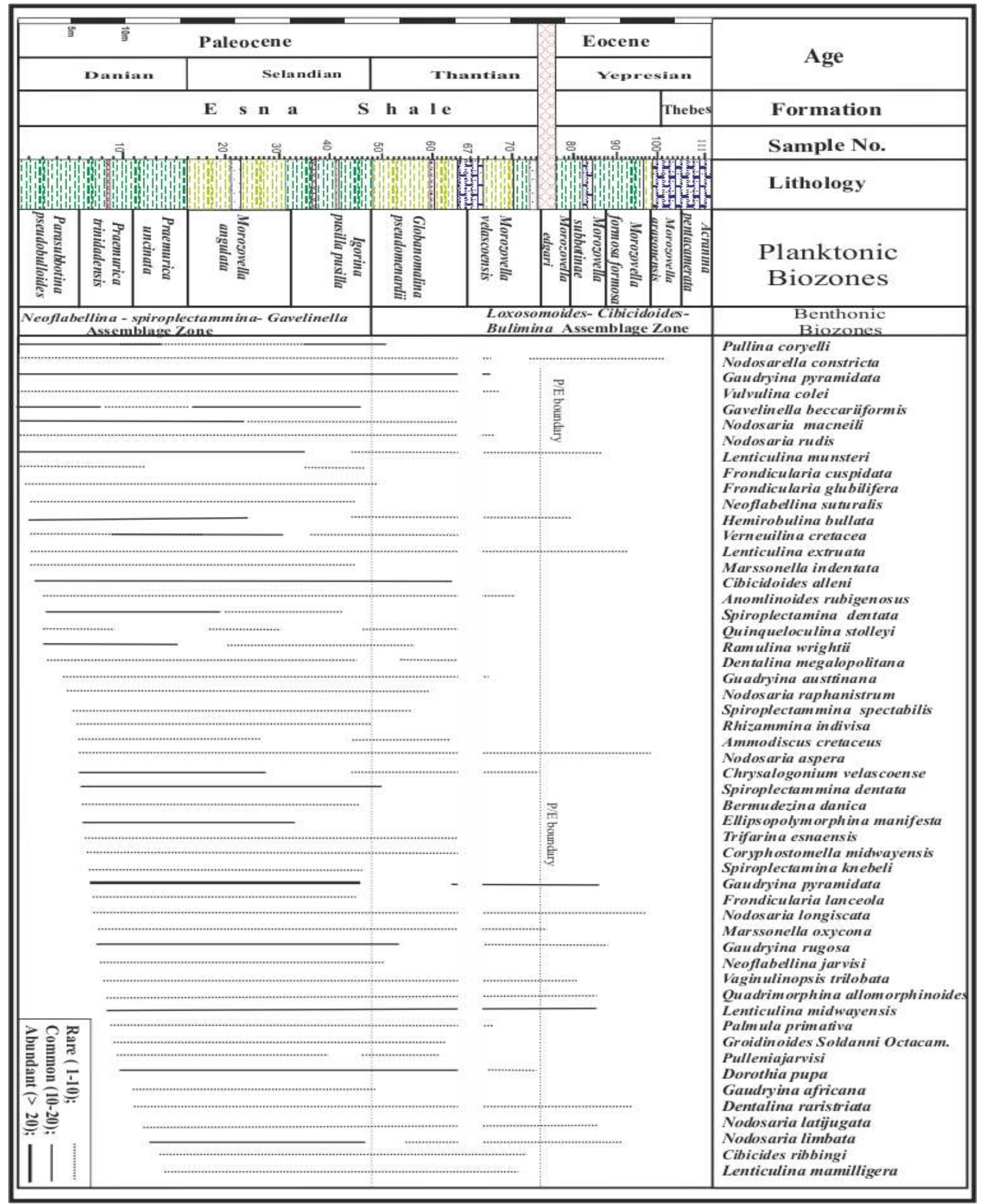

Fig. (5): Biostraigraphic chart of the benthonic foraminiferal species in G. Holet Abu Senna section, northeastern Sinai, Egypt. 


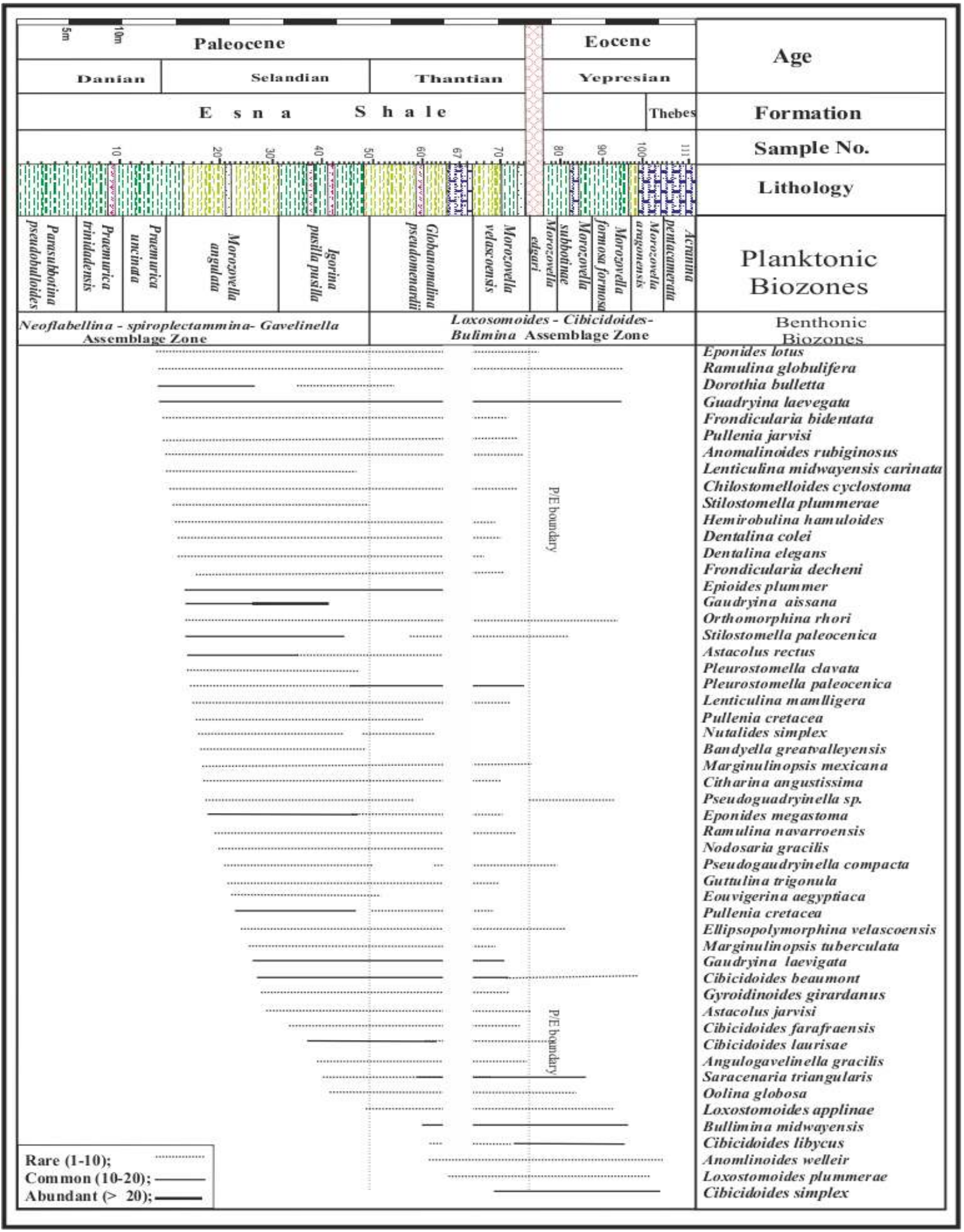

Fig. (5) Continued: Biostraigraphic chart of the benthonic foraminiferal species in G. Holet Abu Senna section, northeastern Sinai, Egypt. 


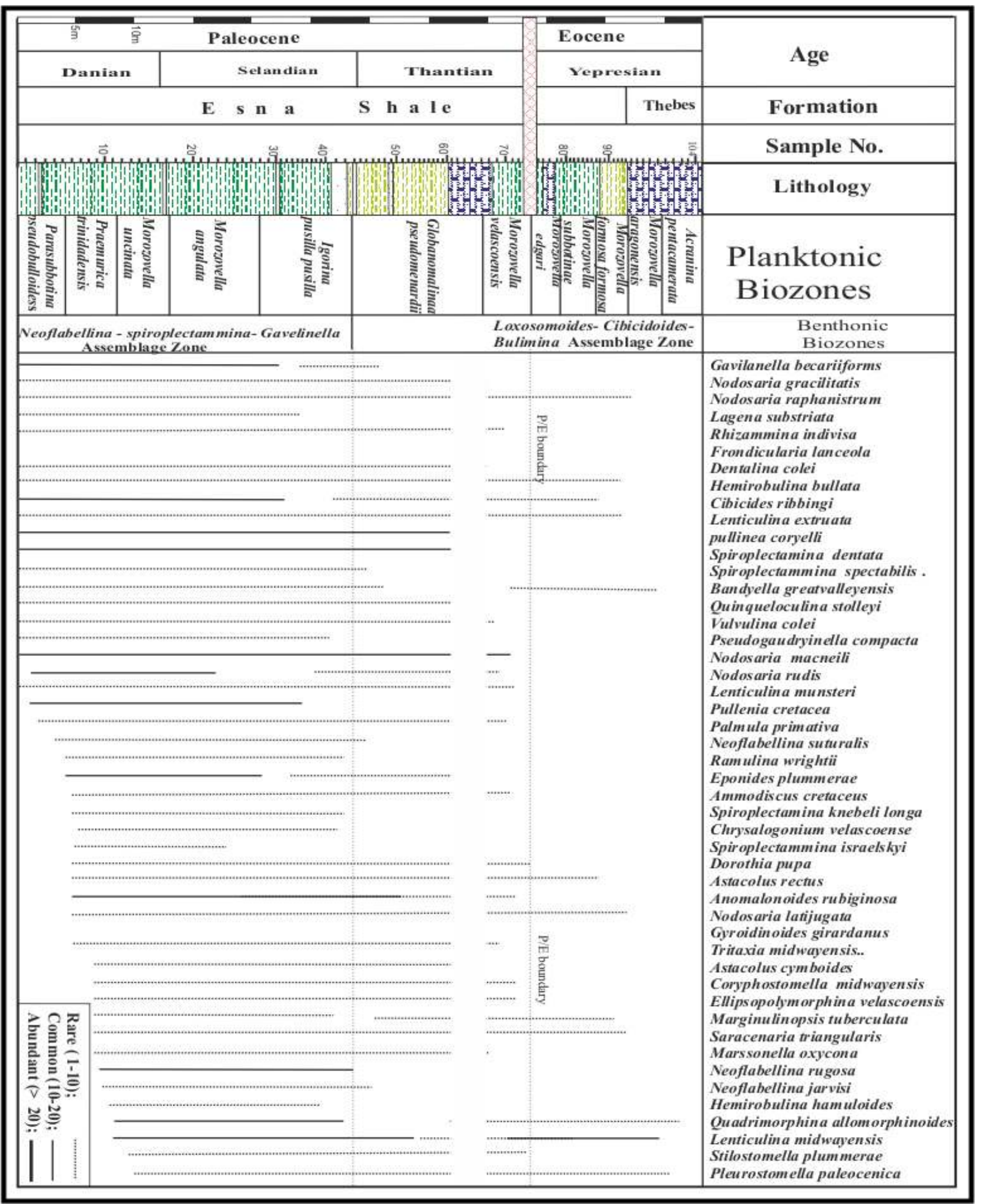

Fig. (6): Biostraigraphic chart of the benthonic foraminiferal species in G. Um Thamalla section, northeastern Sinai, Egypt. 


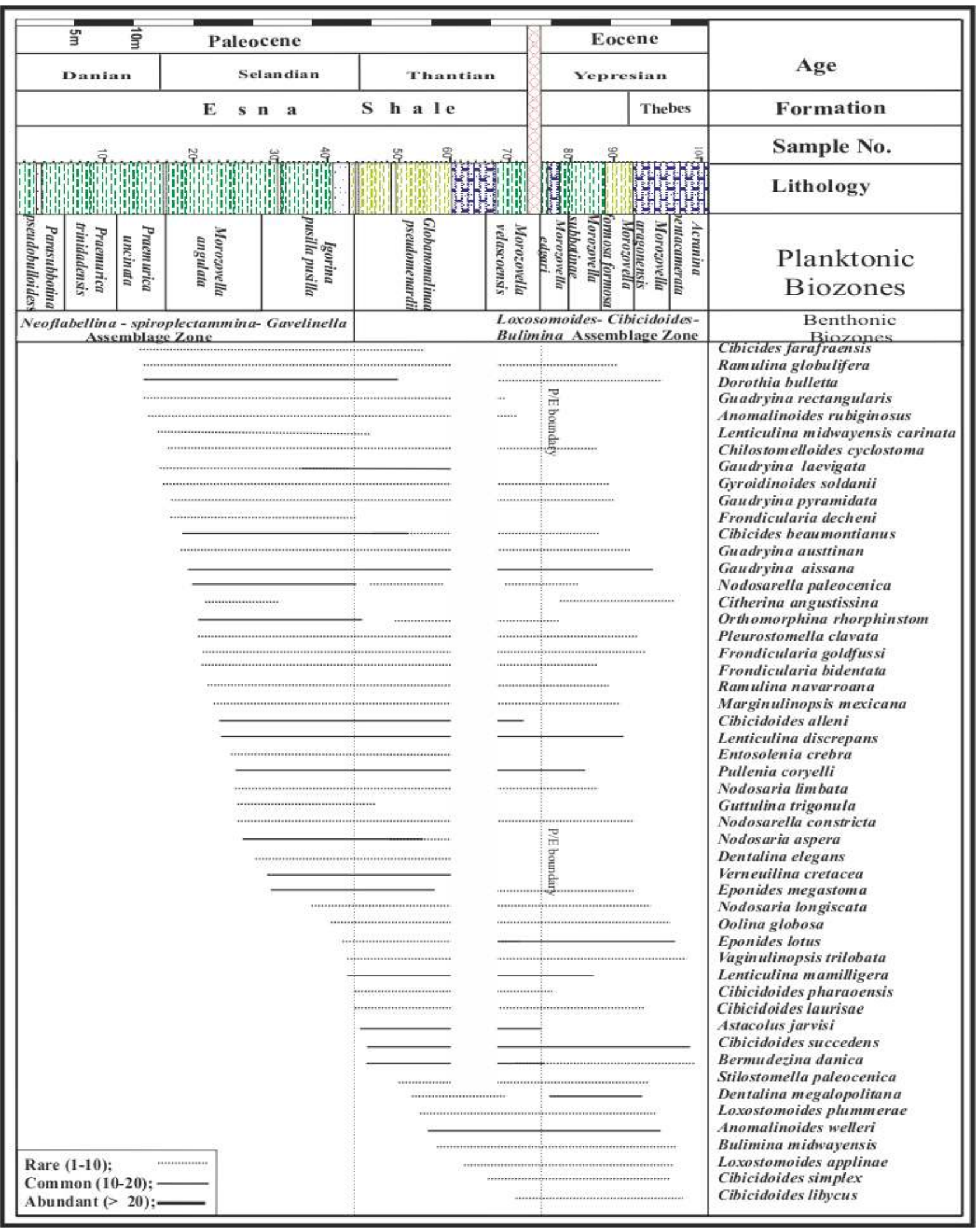

Fig. (6) Continued: Biostraigraphic chart of the benthonic foraminiferal species in G. Um Thamalla section, northeastern Sinai, Egypt. 
Table (1): Statistical analysis of the foraminifera and ostracod species in the Gabal El Qusaima section, as example

Planktonic Foram.

Benthonic Foram.

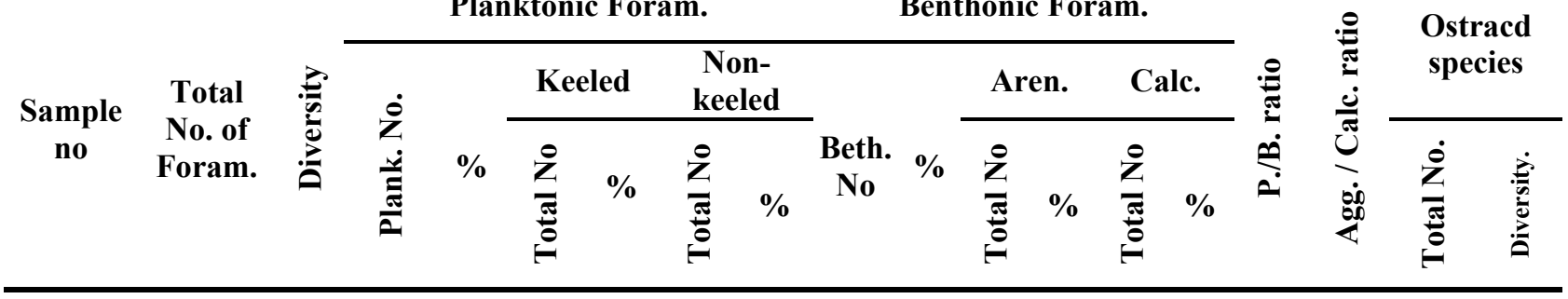

\begin{tabular}{|c|c|c|c|c|c|c|c|c|c|c|c|c|c|c|c|c|c|c|}
\hline 1 & 452 & 16 & 330 & 63 & 0 & 0 & 330 & 100 & 122 & 27 & 13 & 11 & 109 & 89 & 73 & 11 & - & - \\
\hline 2 & 499 & 25 & 339 & 68 & 0 & 0 & 339 & 100 & 160 & 32 & 18 & 11 & 142 & 89 & 68 & 11 & - & - \\
\hline 3 & 420 & 26 & 315 & 75 & 0 & 0 & 315 & 100 & 105 & 25 & 13 & 12 & 92 & 88 & 75 & 12 & 2 & 1 \\
\hline 4 & 413 & 29 & 264 & 64 & 0 & 0 & 264 & 100 & 149 & 36 & 19 & 13 & 130 & 87 & 64 & 13 & 3 & 1 \\
\hline 5 & 563 & 39 & 400 & 71 & 0 & 0 & 400 & 100 & 163 & 29 & 18 & 11 & 145 & 89 & 71 & 11 & - & - \\
\hline 6 & 467 & 49 & 336 & 72 & 7 & 2 & 329 & 98 & 131 & 28 & 16 & 12 & 115 & 88 & 72 & 12 & 4 & 1 \\
\hline 7 & 406 & 53 & 309 & 76 & 37 & 12 & 272 & 88 & 97 & 24 & 15 & 15 & 82 & 85 & 76 & 15 & 2 & 1 \\
\hline 8 & 399 & 55 & 263 & 66 & 21 & 8 & 242 & 92 & 136 & 34 & 20 & 15 & 116 & 85 & 66 & 15 & 3 & 2 \\
\hline 9 & 502 & 56 & 326 & 65 & 36 & 11 & 290 & 89 & 176 & 35 & 19 & 11 & 157 & 89 & 65 & 11 & 2 & 2 \\
\hline 10 & 465 & 60 & 344 & 74 & 48 & 14 & 296 & 86 & 121 & 24 & 13 & 11 & 108 & 89 & 74 & 11 & 1 & 1 \\
\hline 11 & 498 & 65 & 314 & 63 & 41 & 13 & 273 & 87 & 184 & 37 & 22 & 12 & 162 & 88 & 63 & 12 & - & - \\
\hline 12 & 496 & 69 & 293 & 59 & 35 & 12 & 258 & 88 & 203 & 41 & 26 & 13 & 177 & 87 & 59 & 13 & 4 & 2 \\
\hline 13 & 477 & 77 & 291 & 61 & 18 & 6 & 273 & 94 & 186 & 39 & 26 & 14 & 160 & 86 & 61 & 14 & - & - \\
\hline 14 & 478 & 78 & 325 & 68 & 26 & 8 & 299 & 92 & 153 & 32 & 20 & 13 & 133 & 87 & 68 & 13 & 3 & 1 \\
\hline 15 & 456 & 78 & 315 & 69 & 22 & 7 & 293 & 93 & 141 & 31 & 17 & 12 & 124 & 88 & 69 & 12 & 4 & 2 \\
\hline 16 & 503 & 81 & 352 & 70 & 32 & 9 & 320 & 91 & 151 & 30 & 17 & 11 & 134 & 89 & 70 & 11 & 2 & 1 \\
\hline 17 & 522 & 81 & 365 & 70 & 55 & 15 & 310 & 85 & 157 & 30 & 17 & 11 & 140 & 89 & 70 & 11 & 3 & 1 \\
\hline 18 & 566 & 79 & 413 & 73 & 50 & 12 & 363 & 88 & 153 & 37 & 23 & 15 & 130 & 85 & 73 & 15 & - & - \\
\hline 19 & 574 & 80 & 436 & 76 & 153 & 35 & 283 & 65 & 138 & 24 & 35 & 25 & 103 & 75 & 76 & 25 & - & - \\
\hline 20 & 548 & 86 & 344 & 81 & 124 & 36 & 220 & 64 & 204 & 19 & 53 & 26 & 151 & 74 & 81 & 26 & 3 & 1 \\
\hline 21 & 613 & 80 & 423 & 69 & 169 & 40 & 254 & 60 & 190 & 31 & 51 & 27 & 139 & 73 & 69 & 27 & - & - \\
\hline 22 & 566 & 85 & 413 & 73 & 174 & 42 & 239 & 58 & 153 & 27 & 43 & 28 & 110 & 72 & 73 & 28 & 1 & 1 \\
\hline 23 & 548 & 85 & 417 & 76 & 192 & 46 & 225 & 54 & 131 & 24 & 35 & 27 & 96 & 73 & 96 & 27 & 5 & 2 \\
\hline 24 & 516 & 85 & 387 & 75 & 186 & 48 & 201 & 52 & 129 & 25 & 37 & 29 & 92 & 71 & 75 & 29 & - & - \\
\hline 25 & 644 & 87 & 489 & 76 & 255 & 52 & 234 & 48 & 155 & 24 & 17 & 11 & 138 & 89 & 76 & 11 & - & - \\
\hline 26 & 653 & 83 & 536 & 82 & 273 & 51 & 263 & 49 & 117 & 18 & 14 & 12 & 103 & 88 & 82 & 12 & - & - \\
\hline 27 & 612 & 85 & 471 & 77 & 264 & 56 & 207 & 44 & 141 & 23 & 25 & 18 & 116 & 82 & 77 & 18 & 1 & 1 \\
\hline 28 & 325 & 86 & 247 & 76 & 166 & 67 & 81 & 33 & 78 & 24 & 12 & 16 & 64 & 84 & 76 & 12 & 6 & 4 \\
\hline 29 & 263 & 84 & 205 & 78 & 131 & 64 & 74 & 36 & 58 & 22 & 8 & 13 & 50 & 87 & 78 & 8 & 7 & 4 \\
\hline 30 & 159 & 3 & 106 & 67 & 67 & 63 & 39 & 37 & 53 & 33 & 7 & 14 & 46 & 86 & 67 & 7 & - & - \\
\hline 31 & 180 & 73 & 122 & 68 & 87 & 71 & 35 & 29 & 58 & 32 & 10 & 18 & 48 & 82 & 68 & 10 & - & - \\
\hline 32 & 233 & 79 & 161 & 69 & 97 & 60 & 64 & 40 & 72 & 31 & 8 & 11 & 64 & 89 & 69 & 8 & - & - \\
\hline 33 & 718 & 79 & 503 & 70 & 292 & 58 & 211 & 42 & 215 & 30 & 21 & 10 & 194 & 90 & 70 & 10 & 2 & 1 \\
\hline 34 & 719 & 77 & 518 & 72 & 326 & 63 & 192 & 37 & 201 & 28 & 18 & 9 & 183 & 91 & 72 & 9 & 7 & 3 \\
\hline 35 & 755 & 75 & 695 & 79 & 438 & 63 & 257 & 37 & 60 & 21 & 6 & 11 & 54 & 89 & 79 & 11 & 5 & 2 \\
\hline 36 & 685 & 71 & 514 & 75 & 341 & 65 & 173 & 35 & 171 & 25 & 22 & 13 & 149 & 87 & 75 & 13 & 7 & 5 \\
\hline 37 & 405 & 72 & 308 & 76 & 148 & 48 & 160 & 52 & 97 & 24 & 15 & 15 & 82 & 85 & 76 & 15 & - & - \\
\hline 38 & 399 & 70 & 299 & 75 & 135 & 45 & 164 & 55 & 100 & 25 & 13 & 13 & 87 & 87 & 75 & 13 & 4 & 2 \\
\hline 39 & 402 & 69 & 273 & 68 & 107 & 39 & 166 & 61 & 129 & 32 & 15 & 12 & 114 & 88 & 68 & 12 & 3 & 2 \\
\hline 40 & 325 & 16 & 215 & 66 & 77 & 36 & 138 & 64 & 110 & 34 & 18 & 16 & 92 & 84 & 66 & 16 & - & - \\
\hline 41 & - & - & - & - & - & - & - & - & - & - & - & - & - & - & - & - & - & - \\
\hline 42 & - & - & - & - & - & - & - & - & - & - & - & - & - & - & - & - & - & - \\
\hline 43 & - & - & - & - & - & - & - & - & - & - & - & - & - & - & - & - & - & - \\
\hline 44 & - & - & - & - & - & - & - & - & - & - & - & - & - & - & - & - & - & - \\
\hline 45 & 289 & 66 & 234 & 81 & 96 & 41 & 138 & 59 & 55 & 19 & 8 & 14 & 47 & 86 & 81 & 14 & - & - \\
\hline 46 & 365 & 65 & 277 & 76 & 133 & 48 & 144 & 52 & 88 & 24 & 14 & 16 & 74 & 84 & 67 & 16 & 7 & 3 \\
\hline 47 & 582 & 62 & 454 & 78 & 268 & 59 & 186 & 41 & 128 & 22 & 12 & 9 & 116 & 91 & 78 & 9 & 6 & 3 \\
\hline 48 & 648 & 62 & 486 & 75 & 330 & 68 & 156 & 32 & 162 & 25 & 13 & 8 & 149 & 92 & 75 & 8 & 5 & 4 \\
\hline 49 & 647 & 63 & 531 & 82 & 377 & 71 & 154 & 29 & 116 & 18 & 8 & 7 & 108 & 93 & 82 & 7 & 3 & 1 \\
\hline 50 & 689 & 64 & 572 & 83 & 339 & 68 & 233 & 32 & 117 & 17 & 7 & 6 & 110 & 94 & 83 & 6 & 2 & 1 \\
\hline 51 & 730 & 61 & 555 & 76 & 411 & 74 & 144 & 26 & 175 & 24 & 16 & 9 & 159 & 91 & 76 & 9 & 7 & 5 \\
\hline
\end{tabular}


Table (1) Continued: Statistical analysis of the foraminifera and ostracod species in the Gabal El Qusaima section, as example

\begin{tabular}{|c|c|c|c|c|c|c|c|c|c|c|c|c|c|c|c|c|c|c|}
\hline \multirow{3}{*}{$\begin{array}{c}\text { Sample } \\
\text { no }\end{array}$} & \multirow{3}{*}{$\begin{array}{c}\text { Total } \\
\text { No. of } \\
\text { Foram. }\end{array}$} & \multirow{3}{*}{$\begin{array}{l}\stackrel{\overrightarrow{0}}{0} \\
\frac{0}{0} \\
:\end{array}$} & \multicolumn{6}{|c|}{ Planktonic Foram. } & \multicolumn{6}{|c|}{ Benthonic Foram. } & \multirow{3}{*}{ 赵 } & \multirow{3}{*}{ 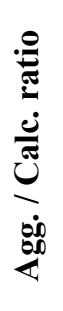 } & \multirow{2}{*}{\multicolumn{2}{|c|}{$\begin{array}{c}\text { Ostracd } \\
\text { species }\end{array}$}} \\
\hline & & & \multirow{2}{*}{$\begin{array}{l}\dot{0} \\
\dot{Z} \\
\dot{I} \\
\dot{E} \\
\dot{a}\end{array}$} & \multirow[b]{2}{*}{$\%$} & \multicolumn{2}{|c|}{ Keeled } & \multicolumn{2}{|c|}{$\begin{array}{c}\text { Non- } \\
\text { keeled }\end{array}$} & \multirow[b]{2}{*}{$\begin{array}{c}\text { Beth. } \\
\text { No }\end{array}$} & \multirow[b]{2}{*}{$\%$} & \multicolumn{2}{|c|}{ Aren. } & \multicolumn{2}{|c|}{ Calc. } & & & & \\
\hline & & & & & $\frac{\stackrel{0}{Z}}{\stackrel{5}{*}}$ & $\%$ & $\frac{\stackrel{0}{Z}}{\stackrel{\pi}{0}}$ & $\%$ & & & $\frac{0}{Z}$ & $\%$ & $\frac{0}{z}$ & $\%$ & & & $\frac{\dot{0}}{\dot{\pi}}$ & 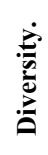 \\
\hline 52 & 755 & 61 & 596 & 79 & 513 & 86 & 83 & 14 & 159 & 21 & 19 & 12 & 140 & 88 & 79 & 12 & 4 & 3 \\
\hline 53 & 723 & 65 & 600 & 83 & 486 & 81 & 114 & 19 & 123 & 17 & 19 & 15 & 104 & 85 & 83 & 15 & - & - \\
\hline 54 & 548 & 61 & 460 & 84 & 368 & 80 & 92 & 20 & 88 & 16 & 14 & 16 & 74 & 84 & 84 & 16 & - & - \\
\hline 55 & 945 & 63 & 832 & 88 & 666 & 80 & 166 & 20 & 113 & 12 & 10 & 9 & 103 & 91 & 88 & 9 & 8 & 5 \\
\hline 56 & 950 & 59 & 817 & 86 & 670 & 82 & 147 & 18 & 133 & 14 & 15 & 11 & 118 & 89 & 86 & 11 & 6 & 4 \\
\hline 57 & 1012 & 48 & 840 & 83 & 722 & 86 & 118 & 14 & 172 & 17 & 24 & 14 & 148 & 86 & 83 & 14 & 3 & 2 \\
\hline 58 & 1003 & 48 & 873 & 87 & 760 & 87 & 113 & 13 & 130 & 13 & 21 & 16 & 109 & 84 & 87 & 16 & 9 & 5 \\
\hline 59 & 1155 & 50 & 901 & 78 & 712 & 79 & 189 & 21 & 254 & 22 & 46 & 18 & 208 & 82 & 78 & 18 & 6 & 3 \\
\hline 60 & 1122 & 47 & 909 & 81 & 709 & 78 & 200 & 22 & 213 & 19 & 36 & 17 & 177 & 83 & 81 & 17 & 4 & 2 \\
\hline 61 & 945 & 46 & 841 & 89 & 664 & 79 & 177 & 21 & 104 & 11 & 12 & 11 & 92 & 89 & 89 & 11 & 7 & 5 \\
\hline 62 & 950 & 45 & 808 & 85 & 646 & 80 & 162 & 20 & 142 & 15 & 11 & 8 & 131 & 92 & 85 & 8 & 9 & 5 \\
\hline 63 & 1012 & 44 & 840 & 83 & 722 & 86 & 118 & 14 & 172 & 17 & 15 & 9 & 157 & 91 & 83 & 9 & 5 & 2 \\
\hline 64 & 1003 & 44 & 812 & 81 & 715 & 88 & 97 & 12 & 191 & 19 & 13 & 7 & 178 & 93 & 81 & 7 & 6 & 3 \\
\hline 65 & 989 & 45 & 791 & 80 & 720 & 91 & 71 & 9 & 198 & 20 & 12 & 6 & 186 & 94 & 80 & 6 & 4 & 2 \\
\hline 66 & 1012 & 43 & 850 & 84 & 782 & 92 & 68 & 8 & 162 & 16 & 11 & 7 & 151 & 93 & 84 & 7 & 7 & 5 \\
\hline 67 & 1003 & 40 & 792 & 79 & 713 & 90 & 79 & 10 & 211 & 21 & 13 & 6 & 198 & 94 & 79 & 6 & 5 & 2 \\
\hline 68 & 1155 & 40 & 1028 & 89 & 956 & 93 & 72 & 7 & 127 & 11 & 12 & 10 & 115 & 90 & 89 & 10 & 4 & 3 \\
\hline 69 & 1122 & 42 & 1021 & 91 & 899 & 88 & 122 & 12 & 101 & 19 & 10 & 10 & 91 & 90 & 91 & 10 & - & - \\
\hline 70 & 950 & 41 & 846 & 89 & 668 & 79 & 178 & 21 & 104 & 11 & 8 & 8 & 96 & 92 & 89 & 8 & 4 & 3 \\
\hline 71 & 1045 & 40 & 910 & 87 & 737 & 81 & 173 & 19 & 135 & 13 & 14 & 10 & 121 & 90 & 87 & 10 & 5 & 2 \\
\hline 72 & 1102 & 39 & 1014 & 92 & 811 & 80 & 203 & 20 & 88 & 8 & 11 & 12 & 77 & 88 & 92 & 12 & 8 & 5 \\
\hline 73 & 1155 & 40 & 1109 & 96 & 954 & 86 & 155 & 14 & 46 & 4 & 7 & 14 & 39 & 86 & 96 & 14 & 10 & 6 \\
\hline 74 & 904 & 41 & 777 & 86 & 629 & 81 & 148 & 19 & 127 & 14 & 21 & 16 & 106 & 84 & 86 & 16 & 15 & 13 \\
\hline 75 & 950 & 38 & 827 & 87 & 496 & 60 & 331 & 40 & 123 & 13 & 16 & 13 & 107 & 87 & 87 & 13 & 5 & 2 \\
\hline 76 & 145 & 42 & 123 & 85 & 64 & 52 & 59 & 48 & 22 & 15 & 3 & 12 & 19 & 88 & 85 & 12 & 11 & 8 \\
\hline 77 & 125 & 41 & 105 & 84 & 50 & 48 & 55 & 52 & 20 & 16 & 4 & 18 & 16 & 82 & 84 & 18 & - & - \\
\hline 78 & 89 & 38 & 80 & 90 & 31 & 39 & 49 & 61 & 9 & 10 & 2 & 19 & 7 & 81 & 90 & 19 & - & - \\
\hline 79 & 112 & 27 & 99 & 88 & 39 & 39 & 60 & 61 & 13 & 12 & 3 & 21 & 10 & 79 & 88 & 21 & 5 & 2 \\
\hline 80 & 102 & 24 & 88 & 86 & 36 & 41 & 52 & 59 & 14 & 14 & 3 & 20 & 11 & 80 & 86 & 20 & - & - \\
\hline 81 & 113 & 13 & 98 & 87 & 37 & 38 & 61 & 62 & 15 & 13 & 4 & 22 & 11 & 78 & 87 & 22 & - & - \\
\hline
\end{tabular}




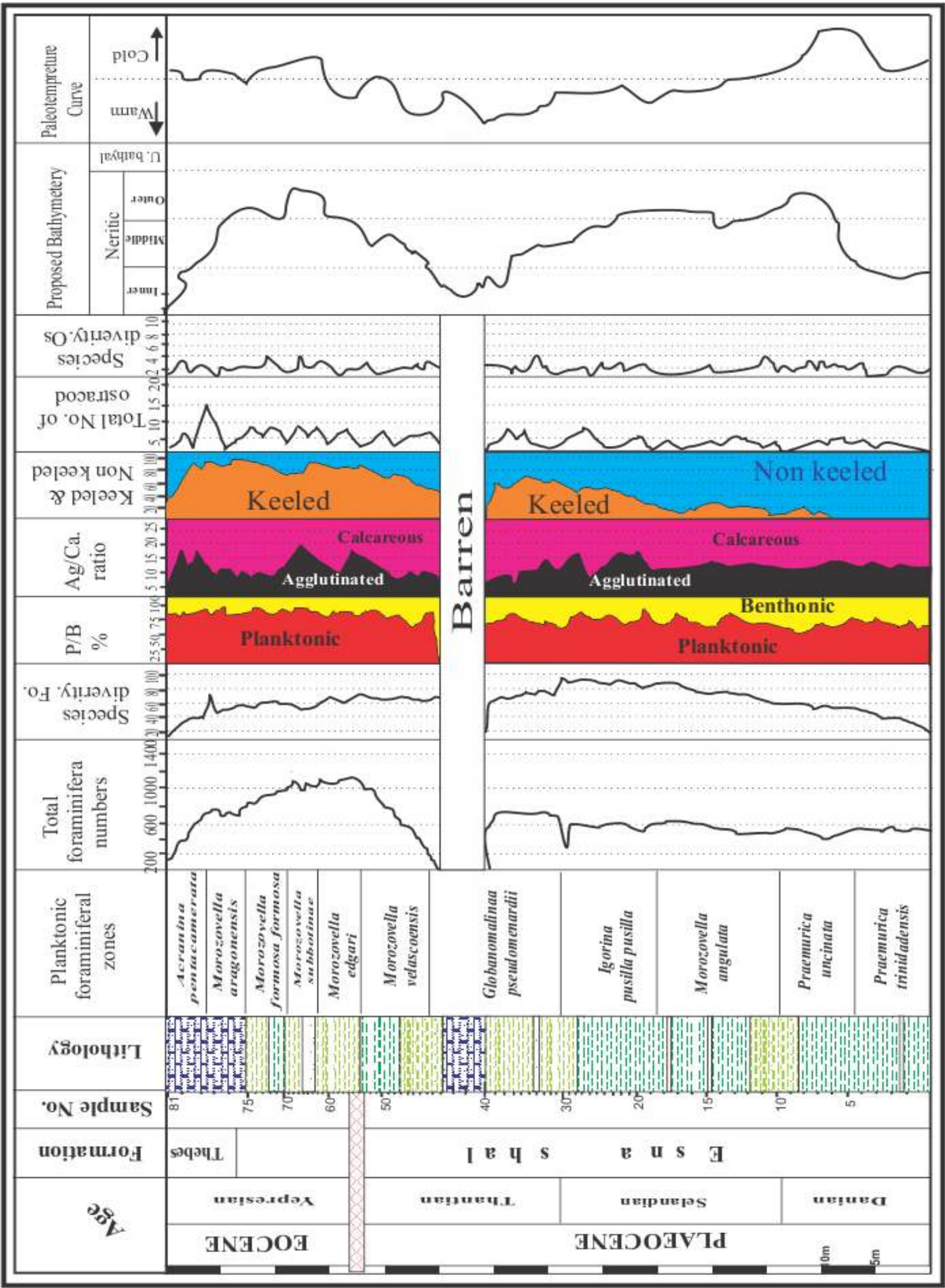

Fig. (7): Paleoecological parameters and paleoenviromental conditions in G. El Qusaima, northeastern Sinai, Egypt, as example 
Plate 1

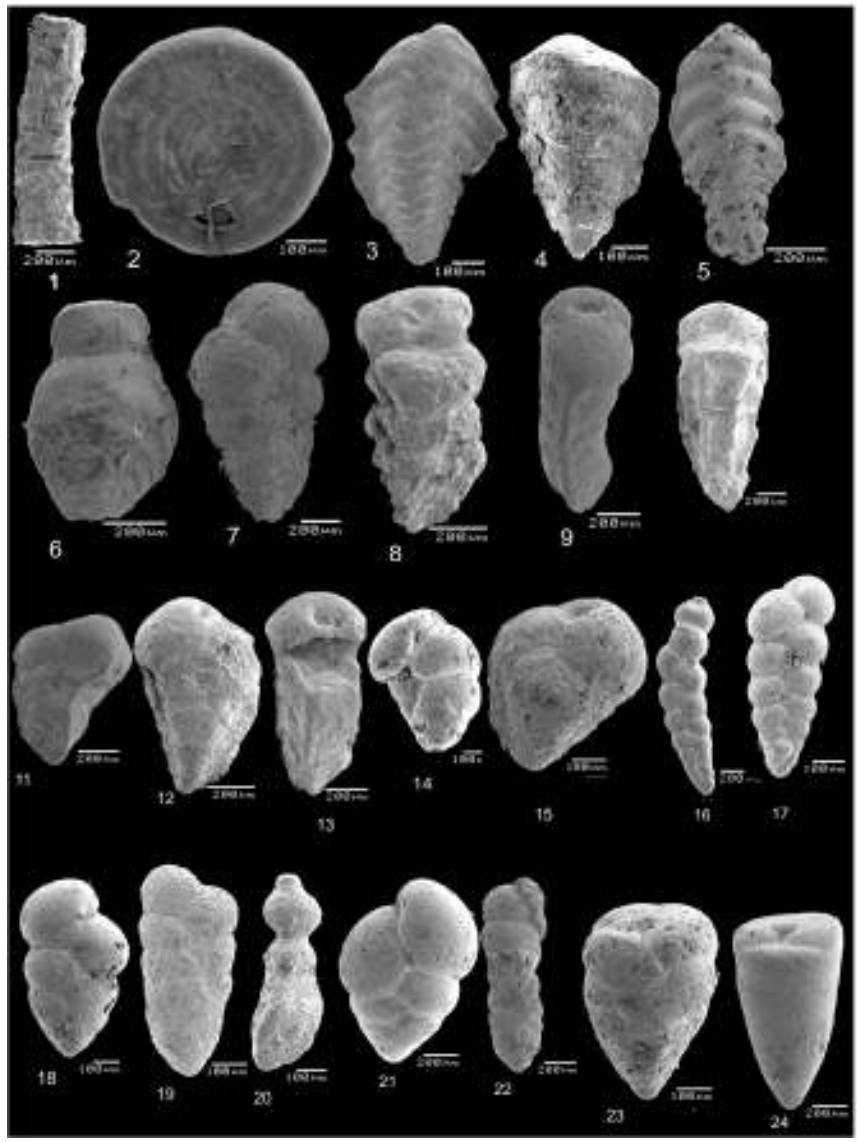

1) Rhizammina indivisa (Brady).

Side view, sample No.91, X60, G. Holet Abu Senna.

2) Ammodiscus cretaceus (Reuss),

Side view, sample No.16,X65 . G.Al Qusaima.

3) Spiroplectamina dentata (Alth)

Side view, sample No.18, X90, G. Holet Abu Senna.

4) Spiroplectamina knebeli (LeRoy ).

Side view, sample No.91, , G. Holet Abu Senna.

5) Spiroplectammina spectabilis (Grzybowsk

Side view sample No.30 ,X75 . G.Al Qusaima.

6) Valvulina colei (Cushman).

Lateral view, sample No. 15, X95, G. Al Qusaima.

7) Gaudryina africana (LeRoy).

Side view, sample No. 34, X60, G. Holet Abu Senna.

8) Gaudryina aissana (Ten Dam\& Sigal).

Apertural view, sample No.34, X90 , G. Holet Abu

Senna.

9) Guadryina austtinana (Cushman).

Apertural view, sample No. 88, X65, G. Holet Abu

Senna.

10) Gaudryina laevigata (Frank).

Apertural view, sample No. 40, X75, G. El Falig.

11) Gaudryina pyramidata (Cushman).

Side view, sample No. 85, X75, G. Holet Abu Senna.

12) Gaudryina rugosa (D Orbigny).

Apertural view, sample No. 12, X100, G. El falig.
13) Pseudogaudryinella compacta. (Ten Dam \& Sigal).

Apertural view, sample No. 18, X110, G. El falig.

14) Pseudogaudryinella sp(Ten Dam \& Sigal).

Apertural view, sample No. 95, X80, G. Holet Abu

Senna.

15) Verneuilina cretacea (Karrer).

-Apertural 1 view, sample No.36 ,X120 . G.Al

Qusaima.

16) Bermudezina danica (Franke)

Side view, sample No.26, X120 . G.Al Qusaima.

17) Bermudezina sp.

Side view, sample No. 103, X75, G. Holet Abu Senna

18) Tritaxia midwayensis (Cushman).

Side View, sample No. 60, X110, G. El Falig.

19) Dorothia bulletta (Carsey).

Side view, sample No. 10, X130, G.Um Thamalla.

20) Pseudoclavulina clavata (Cushman).

Side view, sample No. 41, X65, G. Holet Abu Senna.

22) Marsonella ellisorae (Cushman).

Side view, sample No. 19, X65, G.Um Thamalla.

23) Marssonella indentata (Cushman \& Jarvis).

Apertural view, sample No. 18, X130, G.Um Thamalla

24) Marssonella oxycona (Reuss).

Apertural view, sample No. 18, X65, G. Holet Abu Senna. 


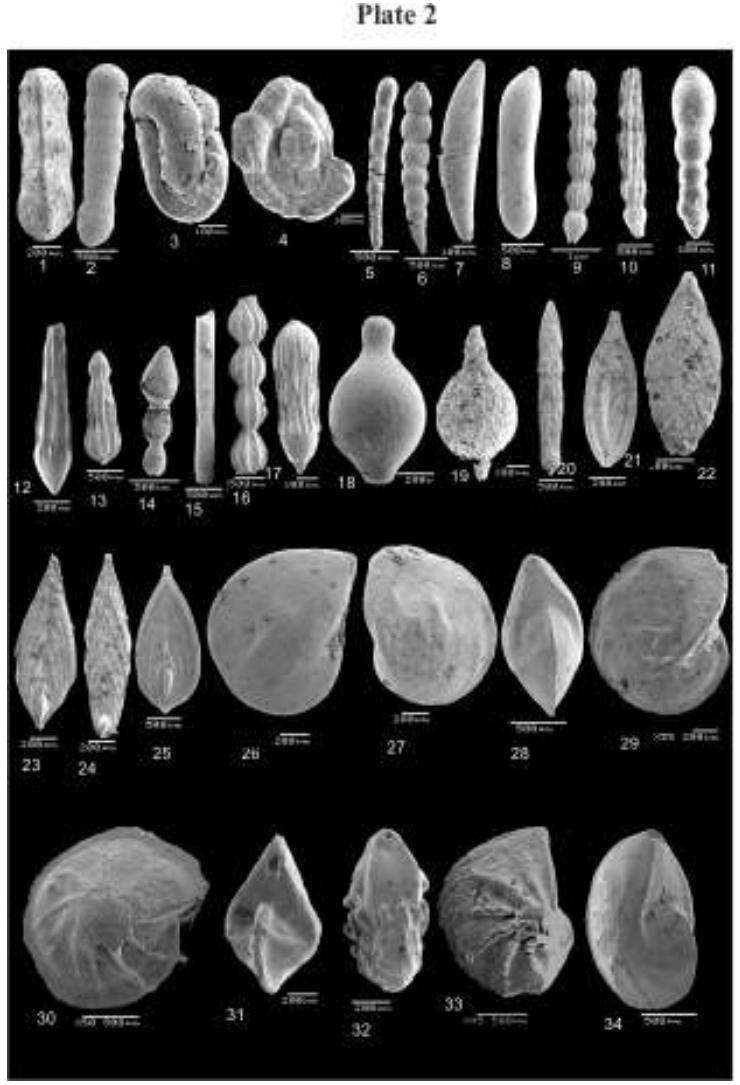

1) Pseudoclavulina globulifera (Ten Dam \& Sigal). Side view, sample No. 91, X40, G. Holet Abu Senna 2) Clavulinoides aspera (Cushman).

Side view, sample No.66,X65 . G.Al Qusaima. 3,4) Quinqueloculina stolleyi (Brotzen). 3-Dorsal view , sample No. 17, X 140 . G. Holet Abu Senna

4-Ventral view, sample No. 8, X140, G. El Falig. 5) Chrysalogonium velascoense (Cushman). Side view, sample No. 66, X 65 . G. Holet Abu Senna 6) Dentalina colei (Cushman \& Dusenbury). Side view, sample No. 73, X40, G. El Falig. 7) Dentalina elegans (D’Orbigny). Side view, sample No. 12, X60, G. El Falig. 8) Dentalina megalopolitana (Reuss).

Side view, sample No. 75, X40, G.Um Thamalla. 9,10) Dentalina raristriata (Chapman).

9-Side view, sample No. 3, X 30 . G. Holet Abu Senna.

10-Side view, sample No. 61, X 30 . G. Holet Abu Senna.

11) Nodosaria aspera (Reuss).

Side view, sample No. 55, X100, G.Um Thamalla

12) Nodosaria gracilitatis (Cushman)

Side view, sample No.3 , X27 . G.Al Qusaima.

13) Nodosaria latejugata (Guembel).

Side view, sample No. 71, X 45 . G. Holet Abu Senna 14) Nodosaria limbata (D'Orbigny).

Side view, sample No. 13, X 45 . G. Holet Abu Senna. 15) Nodosaria longiscata (D'Orbigny).

Side view, sample No.28, X33 . G.Al Qusaima.

16) Nodosaria macneili (Cushman).

Side view , sample No. 13, X 37 . G. Holet Abu Senna 17) Nodosaria raphanistrum (Linne).

Side view, sample No. 55, X 95 . G. Holet Abu Senna
18,19) Nodosaria rudis (D'Orbigny). 18- Side view, sample No. 76, X95, G. El Falig 19- Side view, sample No.82, X 100 . G. Holet Abu Senna.

20) Frondicularia bidentata (Cushman) Side view, sample No. 20, X30, G.Um Thamalla 21) Frondicularia cuspidata (Cushman). Side view, sample No. 50, X85, G.Um Thamalla. 22) Frondicularia decheni (Reuss). Side view, sample No. 32, X45, G. El Falig. 23) Frondicularia goldfussi (Reuss).

Side view, sample No. 71, X60, G. El Falig. 24) Frondicularia lanceola (Reuss).

Side view, sample No.53, X60 . G. Holet Abu Senna.

\section{5) Frondicularia mucronata (Reuss).}

Side view, sample No. 50, X37, G.Um Thamalla. 26-29) Lenticulina discrepans (Reuss).

Side view, sample No. 18, X65 . G. Holet Abu Senna. Side view, sample No. 18, X60 . G. Holet Abu Senna. Apertural view, sample No. 10, X50 . G. Holet Abu Senna.

Side view view sample No.19, X55. G.Al Qusaima. 30,31) Lenticulina extruata (Cushman).

Side view, sample No.10, X50 . G. Holet Abu Senna. Apertural view, sample No. 10, X65 . G. Holet Abu Senna.

32,33) Lenticulina mamilligera (Karre).

Apertural view view sampleNo.36, X45. G.Al

Qusaima.

Peripheral view view sample No.36, X85. G.Al

Qusaima.

34) Lenticulina midwayensis (Plummer).

Peripheral view, sample No. 71, X50, G. El Falig. 


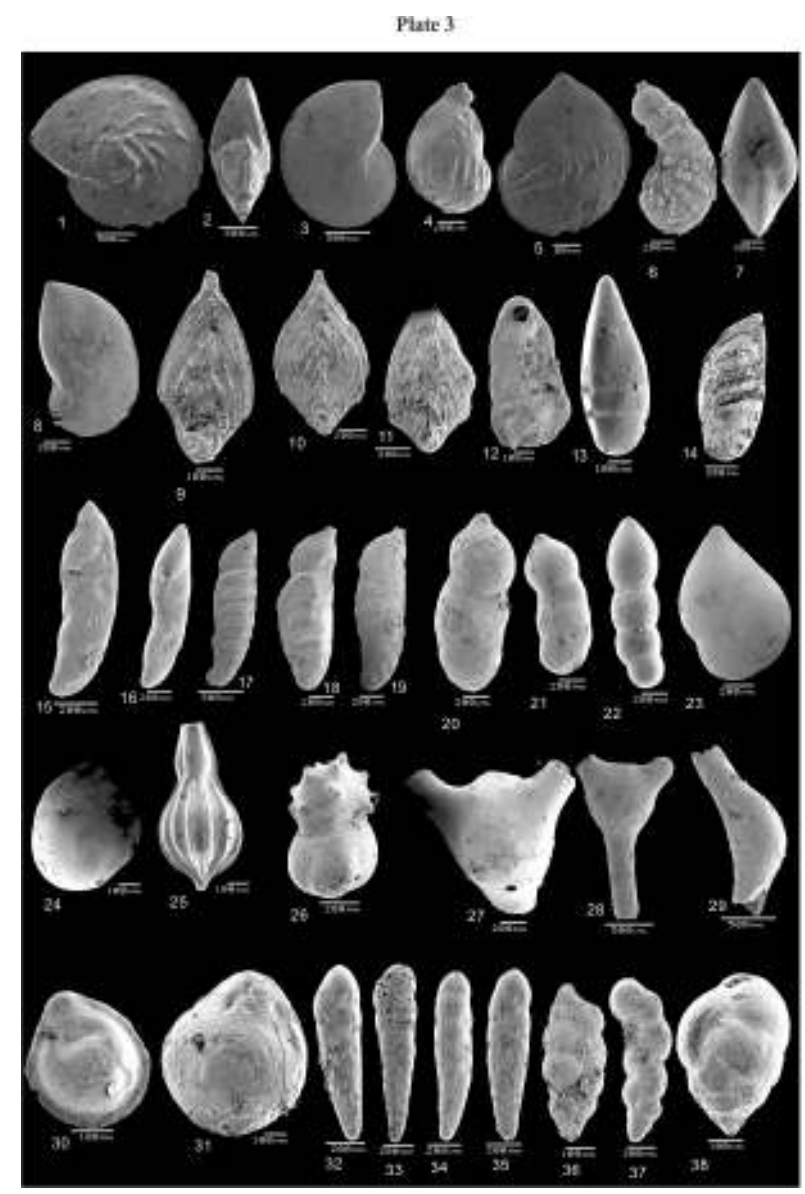

1) Lenticulina midwayensis carinata (Plummer). 1- Side view sample No.19, X43. G.Al Qusaima 2-3) Lenticulina muensteri (Roemer).

2- Peripheral view view sample No.19, X37. G.Al Qusaima. 3- Side view, sample No. 70, X45, G. El Falig.

4,5) Marginulinopsis mexicana alticostata (Cushman \& Barksdale).

4- Side view, sample No.102, X65 . G. Holet Abu Senna.

5- Side view, sample No.102, X85. G. Holet Abu Senna.

6) Marginulinopsis tuberculata (Plummer).

6- Side view, sample No.66, X60 . G. Holet Abu Senna.

7,8) Saracenaria triangularis (D'Orbigny)

7- Apertural view, sample No. 83, X110, G.Um Thamalla.

8- Side view, sample No. 83, X110, G.Um Thamalla.

9) Neoflabellina jarvisi (Cushman).

9- peripheral view sample No.17, X110. G.Al Qusaima.

10) Neoflabellina rugosa (D'Orbigny).

10- Side view, sample No. 83, X65, G.Um Thamalla.

11) Neoflabellina suturalis (Cushman).

11- Side view, sample No. 18, X85, G. El Falig.

12) Palmula primativa (Cushman)

12- Apertural view sample No.60 , X100. G.Al Qusaima

13- Astacolus cymboides (D'Orbigny).

13- Side view, sample No.14, X100 . G. Holet Abu Senna.

14- Astacolus jarvisi (Cushman).

14- Side view, sample No.80, X44 . G. Holet Abu Senna.

15,16) Astacolus rectus (D'Orbigny).

15- Side view, sample No. 45, X90, G. El Falig. 16- Side view, sample No. 45, X60, G. El Falig. 17-19 )Vaginulinopsis trilobata (D'Orbigny).

17- Side view, sample No. 88, X45, G. El Falig.

18- Side view, sample No. 88, X65, G. El Falig.

19- Side view, sample No. 88, X60, G. El Falig.
20-22) Hemirobulina bullata (Reuss)

20- Side view sample No.66, X60. G.Al Qusaima.

21- Side view, sample No.60, X65 . G. Holet Abu Senna.

22- Side view , sample No.10, X65 . G. Holet Abu Senna.

23) Hemirobulina hamuloides (Brotzen).

23- Side view, sample U.M. 25, X70, G.Um Thamalla.

24) Citharina angustissima (Reuss).

24- Side view, sample No. 100, X24, G.Um Thamalla.

25- Lagena substriata (Williamson)

25- Side view sample No.16, X100. G.Al Qusaima.

26) Guttulina trigonula (Reuss).

26-Ventral view, sample No. 23, X90, G. Holet Abu Senna.

27) Ramulina globulifera (Brady).

27- Side view, sample No. 49, X45, G.Um Thamalla.

28 )Ramulina navarroana (Cushman).

28- Side view sample No.34, X60. G.Al Qusaima.

29) Ramulina wrightii (Barnard).

29- Side view, sample No. 36, X50 . G. Holet Abu Senna.

30) Entosolenia crebra (Matthes).

30- Side view , sample No. 25, X190 , G. Al Qusaima

31) Oolina globosa (Montagu).

31- Side view, sample No.72, X100, G. Holet abu Senna. 32,33) Loxostomoides applinae (Plummer).

32- Side view, sample No. 76, X90, G. El Falig

33- Side view, sample No. 76, X70, G. El Falig.

34,35) Loxostomoides plummerae (Cushman).

34- Side view, sample No.82 , X80 . G.Al Qusaima.

35- Side view, sample No.85 , X80 . G.Al Qusaima.

36,37) Eouvigerina aegyptiaca (Nakkady).

36- Side view, sample No.21, X130 . G. Holet abu Senna.

37- Side view, sample No.26, X130 . G. Holet abu Senna.

38) Bulimina midwayensis (Cushman \& Parker).

38- Apertural view sample No.52 , X160 . G.Al Qusaima 


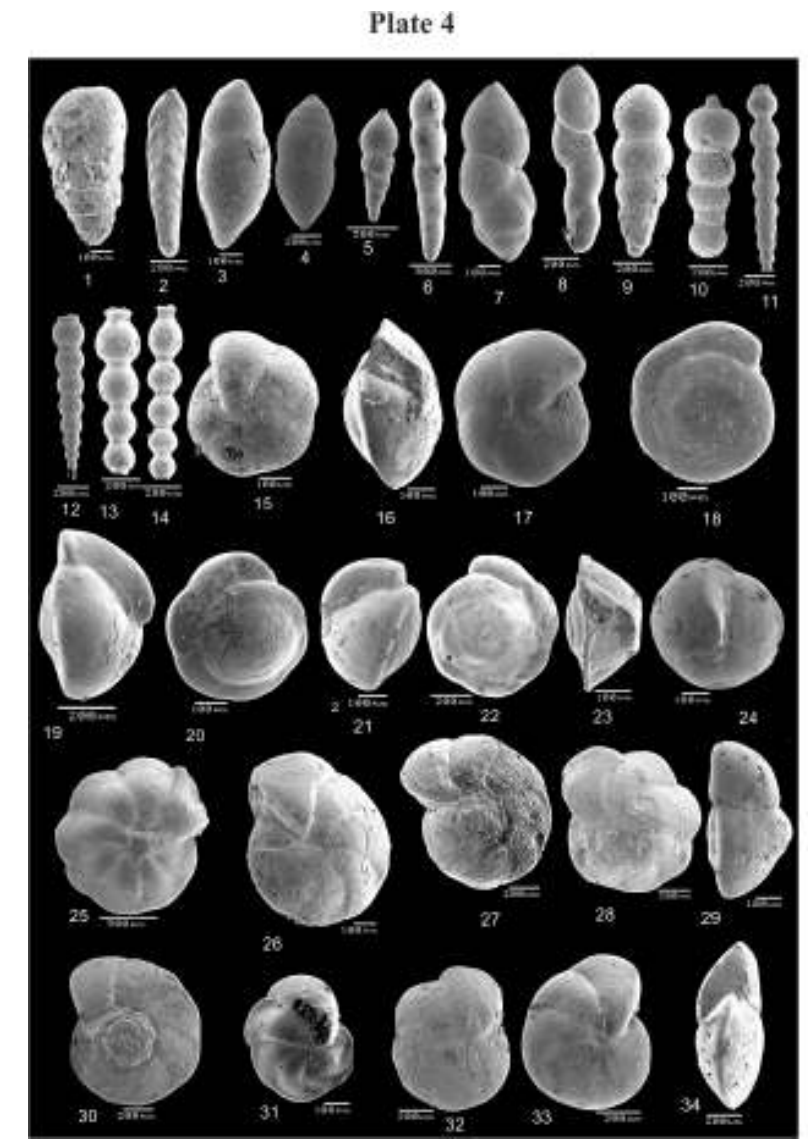

1) Trifarina esnaensis (LeRoy).

1- Side view, sample No. 3, X100, G. El Falig.

2) Coryphostomella telatynensis (Gawor-Biedowa).

2- Lateral view view, sample No. 46, X80, G. El Falig.

3,4) Ellipsopolymorphina velascoensis (Cushman).

3-Side view, sample No.95, X 80 . G. Holet Abu Senna.

5) Nodosarella constricta (Cushman \& Bermudez).

5- Side view, sample No. 16, X 45 . G. Holet Abu Senna.

6) Nodosarella paleocenica (Cushman \& Todd).

6- Side view, sample No. 55, X40, G.Um Thamalla .

7) Pleurostomella clavata (Cushman) .

7-Peripheral view sample No. 18 , X100 . G.Al Qusaima.

8) Pleurostomella paleocenica (Cushman).

8- Peripheral view, sample No. 41, X80, G. El Falig

9) Bandyella greatvalleyensis (Trujillo).

9- Side view, sample No. 56, X45, G.Um Thamall

10) Orthomorphina rhori (Cushman\& Stainforth).

10- Side view, sample No. 43, X60, G. El Falig.

11,12) Stilostomella paleocenica (Cushman \& Todd).

11- Side view, sample No. 62, X 60, G. Holet Abu Senna

12- Side view, sample No. 68, X 70 ,G. Holet Abu Senna

13,14) Stilostomella plummerae (Cushman).

13- Side view, sample No. 22, X60, G. El Falig.

14- Side view, sample No. 23, X60, G. El Falig.

15-17) Eponides megastoma (Grzybowski).

15- Umbilical view, sample No.23, X110, G. El Falig.

16- Peripheral view, sample No. 53, X140, G. Holet abu

Senna.

17- Umbilical view, sample No. 38, X120, G.Um Thamalla.

18,19) Eponides lotus (Schwager).

18- Spiral view, sample No. 70, X100, G. El Falig.
19- Peripheral view, sample No. 16, X95, G. El Falig. 20) Eponides lotus (Schwager).

20- Spiral view , sample No. 22, X140, G. Al Qusaima. 21,22) Eponides plummerae (Cushman).

21- Peripheral view, sample No. 23, X140, G. Holet abu Senna

22- Spiral view, sample No. 23, X90, G. Holet abu Senna. 23,24) Nuttallides simplex (Nuttall).

23- Peripheral view, sample No. 22,X160, G. Holet Abu Senna.

24- Umbilical view, sample No. 22,X120, G. Holet Abu Senna.

25-28) Cibicidoides alleni (Plummer).

25- Umbilical view, sample No. 15, X50 . G. Holet Abu Senna.

26- Umbilical view, sample No. 16, X50 . G. Holet Abu Senna.

27- Spiral view sample No.19, X65. G.Al Qusaima.

28- Umbilical view, sample No. 36, X88 . G. Holet Abu Senna.

29,30) Cibicidoides libycus (LeRoy).

29- Peripheral view, sample No. 88, X50 . G. Holet Abu Senna.

30- Dorsal Spiral view, sample No. 88, X50 . G. Holet Abu Senna.

31) Cibicidoides pharaonis (LeRoy).

31- Umbilical view, sample No. 86, X110, G.Um Thamalla. 32-34) Cibicidoides simplex (Brotzen).

32- Umbilical view, sample No. 79, X75, G. Holet Abu Senna. 33- Umbilical view, sample No. 82, X95, G. Holet Abu Senna. 34-Peripheral view, sample No. 88, X75, G. Holet Abu Senna. 
Plate 5

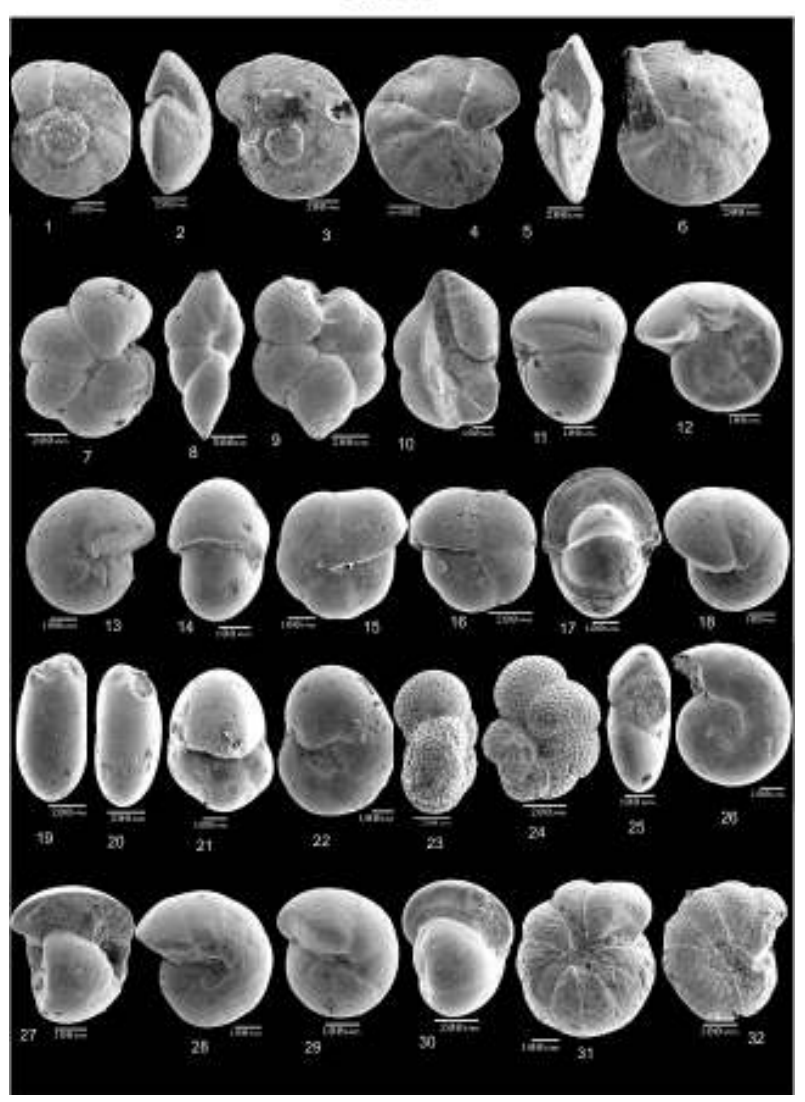

1-3) Cibicidoides succedens (Brotzen).

1- Spiral view, sample No. 78 X80, G.Um Thamalla.

2- Peripheral view, sample No. 78, X85, G.Um Thamalla.

3- Spiral view, sample No. 75, X75, G.Um Thamalla.

4-6) Cibicidoides laurisae ( Mallory).

4- Umbilical view, sample No. 44, X110, G. Al Qusaima.

5- Peripheral view, sample No. 49, X45, G.Um Thamalla 6- Umbilical view, sample No. 49, X45, G.Um Thamalla

7) Cibicides beaumontianus (D'orbigny)

7- Umbilical view, sample No. 36, X90, G. El Falig. 8-10) Cibicides ribbingi (Brotzen).

8- Ventral view, sample No. 22, X45, G. El Falig. 9- Ventral view view sample No.30, X80. G.Al Qusaima 10- Dorsal view, sample No. 24, X100, G. El Falig.

11-13) Pullenia coryelli (White).

11- Peripherall view, sample No. 23, X150, G. Um Thamalla.

12- Spiral view, sample No. 23, X150, G. Um Thamalla.

13- Umbilical view, sample No. 14, X120, G. Al Qusaima.

14-16) Pullenia cretacea (Cushman).

14- Peripheral view , sample No. 18, X95, G. Al Qusaima

15- Ventral view, sample No. 18, X95, G. Al Qusaima

16- Ventral view, sample No. 23, X120, G. Holet abu Senna

17,18) Pullenia jarvisi (Cushman).

17- Peripheral view, sample No. 28, X140, G. Holet abu Senna.
18- Ventral view, sample No. 23, X120, G. Holet abu Senna 19,20) Chilostomelloides cyclostoma (Rzehak).

19- Side view sample No.18 , X85. G.Al Qusaima 20- Side view sample No.18, X85. G.Al Qusaima. 21,22) Quadrimorphina allomorphinoides (Reuss ).

21- Ventral view, sample No. 18, X100, G. Holet Abu Senna. 22- Ventral view, sample No. 18, X120, G. Holet Abu Senna. 23-24) Anomalinoides rubiginosus (Cushman). 23- Peripheral view, sample No. 44, X110, G. Al Qusaima. 24- Ventral view, sample No. 36, X90, G. Holet Abu Senna. 25,26) Anomalinoides welleri (Plummer).

25- Peripheral view, sample No. 13, X110, G.Um Thamalla. 26- Spiral view, sample No. 13, X130, G.Um Thamalla. 27,28) Gyroidinoides girardanus (Reuss).

27- Side view , sample No. 22, X130 , G. Holet Abu Senna 28- Ventral view, sample No. 16, X150, G. El Falig.

29,30) Gyroidinoides soldanii octocameratus (Cushman \& Hanna).

29- Ventral view , sample No. 55, X140, G. Holet Abu Senna. 30- Peripheral view, sample No. 55, X90, G. Holet Abu Senna.

31) Angulogavelinella gracilis (Marsson).

31- Ventral view, sample No. 54, X110, G. Al Qusaima.

32) Gavelinella beccariiformis (White).

32- Ventral view , sample No. 35, X70, G. Holet Abu Senna 


\section{CONCLUSION}

More than 109 benthonic foraminifera species are recorded from the exposed rock unites (Esna and Thebes formations).in the studied sections. Their vertical distribution are shown and most of them are photographed with SEM and illustrated in 5 plates. The investigation of the foraminiferal content has led to the recognition of two assemblage zone. Their age determination depend mainly on the equivalent planktonic foraminifera content. The statistical analysis is carried out in details to determine the paleoenviromental parameters, base on $\mathrm{P} / \mathrm{B}$ Ratio, Agg./Calc. Ratio. Could be divided paleoenvironmental events of the studied sections into three biofacies Biofacies 1 (before PETM), Biofacies 2 (during PETM) and Biofacies 3 (After PETM). The Esna Shale was deposited in a middle neritic to upper bathyal environment and Thebes Formation was also deposited in progressively shallowing middle neritic environment.

\section{REFERENCES}

Alegret, L., Ortiz, S., Arenillas, I. \& Molina, E. (2009): Palaeoenvironmental turnover across the Palaeocene/Eocene boundary at the stratotype section in Dababiya (Egypt) based on benthic foraminifera. Terra. Nova 17: 526-536.

Ayyad, S. N. (2001):Upper Cretaceous LowerTertiary planktonic foraminifera from Bir Safra area, Southern Sinai, Egypt. The second Conf. on Ecology, Damietta, 2001.

Ayyad, S. N. (2003): Paleoecology and Paleobathymetry of the upper MaastrichtianMiddle Eocene succession at Nprtheastern Sinai, Egypt. The third International Conf.e on the geol. of Africa, Assiut, 7-9 December.75-83. Egypt. Assiut -Egypt.

Bains, S., Norris, R. D., Corfield, R. M. \& Faul, K. L.( 2000): Termination of global warmith at the Paleocene / Eocene boundary through productivity feedback. Nature 407:171-174.

Berggren, W. A. \& Aubert, J. (1975): Paleocene benthonic foraminifera biostratigraphy paleobiogeography and paleoecology of the Atlantic -Teethhyanregion: Midway type fauna. Paleogeogra. Paleoclimatol. Paleoecolo. Netherland:El Sevier. 18:73-192.

Dickens, G. R. (2003): Hydrocarbon -driven warming. Nature(429): 513-515.

El-Nady, H. E. A. (2001a): Late Cretaceous - Early Tertiary agglutinated foraminifera and their paleoecology in the Gabal El -Ghorra section, northern Sinai, Egypt. Revue paleobiol, Geneve (Join 2002) 21(1): 413-437.

El-Nady, H. E. A. (2001b): Late Cretaceous planktonic foraminifera biostrtaigraphy and paleobathometery in northeastern Sinai, Egypt, $11 \mathrm{p}, 345-383$.

El-Nady, H. E. A. (2005): The Impact of Paleocene / Eocene boundary events in northern Sinai, Egypt; Planktonic foraminiferal biostratigraphy and faunal turnovers. Revue. paleobiol. Geneve, 24(1): $1-16$.
El-Nady, H. \& Shahin, A. (2001): Planktonic foraminifera biostratigraphy and paleobathymetry of the Late Cretaceous - Early Tertiary succession at Northern Siani, Egypt. Jorn. Paleont., Vol. 1, :193-227.

El-Nady, H. E. A, Morsi, A. M. \& Abdalla, H. F. (2008): Integrated Biostratigraphy, Paleoecology and inter-regional correlation with sea levelchanges of the Cretaceous deposits at Gabal El Halal area, Northern Sinai ,Egypt, based on planktonic foraminifera $.4^{\text {th }}$ international Conf .on the Geol. of the Tethys Cairo. Nov. 2008, P. 99-128.

Faris, M., Ayyad, S. N., El Nahass, H. A. \& Wosabi, A.(2005): Integrated planktonic foraminifera and calcareous nannofossils biostratigraphy of the Upper Cretaceous -lowerEocene formation, west central Sinai, Egypt. Proceeding of the $4^{\text {th }}$ international Conf. on the Geol. of Africa, Asssiut, Egypt, 2: 769-791.

Gibbson, S.J., Bown, T. J., Zachos, J. C., \& Bybell, L. M. (2006): Shelf and open-ocean calcareous phytoplankton assemblages across the Paleocene - Eocene Thermal Maximum: implications for global productivity gradients. Geol., 34: 233236.

Hallock, P. (1985): Why are larger foraminifera large? Paleobiology 11, 195-208.

Kaiho, K., (1994a): Benthic foraminiferal dissolvedoxygen index and dissolved-oxygen levels in the modern ocean. Geol. 22: 719-722.

Kaiho, K., (1994b). Planktonic and benthic foraminiferal extinction events during the last 100 m.y. Palaeogeogr. Palaeoclimato. Palaeoecol. 111: 45-71.

Kaiho, K. (1998): Global climatic forcing of deep-sea benthic foraminiferal test size during the past 120m.y. Geol. 26: 491-494.

Kaiho, K. (1999): Evolution in the test size of deep-sea benthic foraminifera

during the past 120 m.y. Marine Micropaleontol. 37: 53-65.

Kaiho, K., Takeda, K., Petrizzo, M. R. \& Zachos, J. C. (2006): Anomalous shifts in tropical Pacific planktonic and benthic foraminiferal test size during the Paleocene- Eocene Thermal maximum. Palaeogeog. Palaeoclimatol. Palaeoecol. Italy, 237: 456-464.

Kennett, J. P. \& Stott, L. D. (1990): Proteus and protoOceanus; Paleogene ocean as revealed from antarctic stable isotopic results. Proc. ODP. Sci. Results 113: 865-879.

Luning, S. H., Kuss, J., Bachmann, M., Marzouk, A. M. \& Moursi, A. M.(1998): Sedimentary response to basin inversion: Middle Cretaceous - Early Tertiary pre- to syndeformational deposition at the Areif El Naqa anticline. Northern Sinai, Egypt. Facies, Erlangen.38: 103-136, pls. 3537. fig. 12.

Luterbacher, H. P. (1964): Studies in some Globorotalia from the Paleogene and lower Eocene of the Central Appennines. Eclogae Helvetiae, 57(2): 631-730. 
Luterbacher, H. P. (1975a): Paleocene and early Eocene planktonic foraminifera Leg 31 Deep Sea Drilling Project. In Larson. R. L., Moberly, R.,et al., 1975. Initial Results of the Deep Sea Drilling Preject.Vol. 32. Washington (U.S.Government Printing Office): 725-728.

Luterbacher, H. P. (1975b): Planktonic foramimfera of the Paleocene and Early Eocene, Possagno section. Schweizerische Palaontologische Abhandlungen. 97: 57-67.

Mancini, E. A. (1984): Biostraligraphy of Paleocene strata in southwestern Alabama, Micropaleontol. 30 (3): 263-291.

Marzouk, A. M. \& Lunino, S.(1998): Comparative biostraligraphy of calcareous nannofossils and planktunic foraminifcra in the Palcocenc of Eastern Sinai, Egypt. N. .Jb. Geol. Palaant., Stuttgart, 207 (1): 77-105.

Monechi, S., Angori, E., \& Speijer, R. P. (2000a): Upper Paleocene biostratigraphy in the Mediterranean region; Zonal merkers, diachronism, and preservational problems. Geol. Soci. of Sweden, (GFF) 122:108-110.

Monechi, S., Angori, E. \&Von Salis, K. (2000b): Calcareous nannofossilis turnover around the Paleocene-Eocene transition at Alamedilla (Southern Spain). Bull. de la Soci. Geo. de France, 171(4):477-489.France.

Morkhoven, E. P. C., Berggern, W. A. \& Edward, A. S. (1986): Cenozoic cosmopolitan deep - sea benthic foraminifera. Bull. Center. Resh. Explor. Prod. Elf-Aquitiane, Mem., 11: 1-421, 8text-figs. 1, table, 126 pls., Appendix, 1-3: Pau.

Morsi. A. M. \& Faris, M., Zalat, A. \& Salem, R. F. M. (2008): Maastrichtian Early Eocene ostracoda from West Centarl Sinai, Egypt - taxonomy, biostratigraphy, paleoecology and paleobiogeography. Revue de Paleobiol. 27(1): 159 - 189.Geneve.

Murray, J. W. (1976) - A method determining proximity of the marginal seas to an ocean. Marine Geol. 22: 103-119.

Nassif. M. S., A. Sadik, and Hassan H. F.(2008): Paleobathymetery and sea level oscillation of some Paleogene rocks across Sinai, Egypt. Fourth Internatio. Conf. on the Geo. Of the Teth. Cairo Univeirist. Nov. 2008. P. 41-66

Obaidalla, N. A. (1999): Planktonic foraminiferal biostratlgraphy of the Paleocene/Eocene boundary transition in south-western Sinai, Egypt. In: Soliman, H.A., Ouda, K.A.K. (Eds.), Symposium on Late Paleocene-Early Eocene events from North Africa to the Middle East wtthin the $1^{\text {st }}$ International Conference on the Geology of Africa. Fac.of Sci. Univ. of Assiut, Egypt. pp. 31-37.

Oreshkina, T. V. \& Oberhansli, H. (2003): DiaTom turnover in the early Paleogene diatomite of the .Sengiley section. Middle Povolzhie, Russia aresponse to the initial Eocene thermal maximum. Boulder, Colorado. Geol. Soci. of America, Speci. paper, vol. 369: 169-180
Parrish, J. T. \& Curtis, R. L. (1982): Atmospheric circulation, upwelling and organic rich rochs in Mesozoic and Cenozoic Eras. Paleogeography, paleoclimatol. Paleoecol. 40: 31-66.

Phleger, F. B. \& Souter, A. (1973): Production of benthonic foraminifera in the three east pacifice oxygen minima. Micropaleontol. 19: 110-115

Röhl, U., Westerhold, T., Bralower\& Zachos, J. C. (2007): On the duration of the Paleocene-Eocene thermal maximum (PETM). Geochemis. Geophys. Geosy. 8: Q12002, doi: 10.1029/2007GC001784.

Salis. K., Vonquda, K. H., Saad, M., Tantawy, A. A. \& Bernascom, S. (1998): Calcareous nannofossils, foraminifera and isotopes studies from the $\mathrm{P} / \mathrm{E}$ boundary sections in Egypt. In: La Limite Paleocene Eocene en Europe: Evemements et correlations (Abstract). -Univ. Paul-SabatierToulouse III. Lab. De Geol. Sediment. Paleontol.. Strata, Ser.,9: 113-115.

Shahin, A. (1992a): Contribution to the foraminiferal biostratigraphy and paleobathymetry of the Late Cretaceous and Early Tertiary in Western Central Sinai, Egypt. Reve. de. Micropaleontol.,35 (2): 157-175.

Shahin, A. (1992b): Planktonic foraminiferal biostratigraphy and paleobathymetry of die subsurface Upper Cretaceous sequence in the Bardwill-1 well, Northern Sinai, Egypt. Mans. Sci. Bull., 9 (2): 35-67.

Shahin, A. (1998): Tertiary Planktonic foraminiferal biostratigraphy and paleobathymetry of Gabal Withr Southwestern Sinai, Egypt. N. Jb. Geol. Paleont.,209(3): 323-348.

Shahin, A. (2001): Mass extinction and binevents across the Paleocene - Eocene boundary in the Western Sinai, Egypt. N. Jb. Geol. Paleontol. Mh., Stuttgart, (I): I-20, 8 figs.

Shackleton, N. J. \& Hall, M. A. (1985a): Carbon isotope data from leg 74 sediments. InMoore, Rabinowitze, P.D. et al., (EDS). Intial Reports of the deep sea Drilling project. United state Government printing office, Washington, DC, 74:613-619.

Shackleton, N. J. \& Hall, M. A. (1985b): Paleotempearature history of the Cenozoic and the initiation of Antaractic glaciation: Oxygen and carbon istope analysis in deep sea drilling project Sites 277, 279, and 281. In Kennett, J. P. Houtz, R. E. et al., (EDS). Initial Reports of the deep sea Drilling project. United state Government printing office, Washington, DC, 29:743-756.

Speijer, R. P. \&Van Der Zwan, G. J. (1994): The differential effect of the Paleocene- Eocene boundaiy events-: Extinction and survivorship in shallow to deep- water Egyptian benthic foraminiferal asssemblages - Geol. .124:121168

Stott, L. P., Kennett, J. P., Shackleton, N. J. \& Corfield, R. M. (1991): The evolution of Antarctic surface natures during the Paleogene: inferences from the stable isotopic composition of planktonic 
foraminifers, ODP Leg 113. In: Barker, P.F., Kennett, J.P., et al. (Eds.), Proc. ODP, Sci. Results, vol. 113. Ocean Drilling Program, College Station, TX, pp. 849-864.

Tjalsma, R, C. \& Lohmann, G. P. (1983) - PaleoceneEocene bathyal and abyssal benthic foraminifera from the Atlantic Ocean. Micropaleontol. Spec. Public. 4: 90 pp.

Thomas, E. (1990): Biogeography of the late Paleocene benthic foraminiferal extinction. In: M. - p. Aubry, s. G. Lucas \& w. A. Berggren (eds). Late Paleocene - Early Eocene Climatic and Biotic Events in the Marine and Terrestrial Records. Columbia Univ. Press, New York, 214-243.

Thomas, E. \& Shackleton, N. J. (1996): The PaleoceneEocene benthonic foraminiferal extinction and stable isotope anomalies. In: Knox. R.W.O'B., Corfield, R. and Dunay, R. (eds.). Correlation of the Early Paleogene in northwest Europe, Geol. Soc., Spec. Publ., 101:401-441.

Thomas, D. J., Zachos, J. C., Bralower, T. J., Thomas, E. \& Bohaty, S. (2002): Warming the fuel for the fire: Evidence for the thermal dissociation of methane hydrate during the Paleocene - Eocene Thermal Maximum. Geol. 30: 1067-1070.

Tremolada, F.\& Bralower, T. J.(2004): nannofossil assemblage fluctuations during the PaleoceneEocene Thermal Maximum at Sites 213 (Indian Ocean) and 401 (North Atlantic Ocean): paleoceanogr. Implicat. Marine Micropaleontol. 52: 107-116. 